\title{
HOMOLOGICAL TYPE OF GEOMETRIC TRANSITIONS
}

\author{
MICHELE ROSSI
}

\begin{abstract}
The present paper gives an account and quantifies the change in topology induced by small and type II geometric transitions, by introducing the notion of the homological type of a geometric transition. The obtained results agree with, and go further than, most results and estimates, given to date by several authors, both in mathematical and physical literature.
\end{abstract}

\section{Contents}

Introduction

1. Geometric transitions

2. Local analysis of an isolated singularity

3. Homological type of a conifold transition

4. Homological type of a small geometric transition

4.1. Homology change induced by a small geometric transition 13

5. Homological type of type II geometric transition $\quad 19$

5.1. Normal exceptional divisor 20

5.2. Non-normal exceptional divisor 21

5.3. Homology change induced by a type II geometric transition 22

5.4. The normal and rational case $\quad 23$

5.5. The elliptic case 30

5.6. The non-normal case 34

References 34

\section{INTRODUCTION}

A geometric transition (g.t.) between two Calabi-Yau threefolds $Y$ and $\widetilde{Y}$ (see Definition 1.1) is the process $T(Y, \bar{Y}, \widetilde{Y})$ obtained by "composing" a birational contraction $\phi: Y \rightarrow \bar{Y}$, to a normal threefold $\bar{Y}$, with a complex smoothing (see Definition 1.4). A g.t. is a useful tool for connecting to each other topologically distinct Calabi-Yau threefolds. This feature is probably the main source of interest in the study of g.t.'s both in mathematics and physics.

In mathematics, the story goes back to deep speculations due to H. Clemens $[6$, R. Friedman [10, F. Hirzebruch [19, J. Werner [53] and M. Reid [41]. In fact, the

This work has been developed despite the effects of the Italian law 133/08 (http://groups.google.it/group/scienceaction). This law drastically reduces public funds to public Italian universities, which is particularly dangerous for free scientific research, and it will prevent young researchers from getting a position, either temporary or tenured, in Italy. The author is protesting against this law to obtain its repeal. 
huge multitude of known topologically distinct Calabi-Yau threefolds makes any concept of moduli space for them immediately wildly reducible. This unpleasant fact dramatically clashes with the well known irreducibility of moduli spaces of both elliptic curves and K3 surfaces, which are the lower dimensional analogues of Calabi-Yau threefolds. Actually, Reid (op. cit.) underlines that, at the beginning (in the forties), the moduli space of (algebraic) K3 surfaces seemed to F. Enriques to be a 19-dimensional variety admitting a countable number of irreducible components, $\mathcal{M}_{g}$, one for each value of the sectional genus, $g \geq 3[9]$. Twenty years later K. Kodaira 24] was able to recover a 20-dimensional irreducible moduli space, $\mathcal{M}$, for K3 surfaces by leaving the algebraic category to work in the larger category of analytic, compact, Kähler varieties, and discovering that the generic K3 surface is an analytic non-algebraic complex variety: in particular the moduli space $\mathcal{M}^{\text {alg }}=$ $\bigcup_{g} \mathcal{M}_{g}$ of algebraic K3's embeds in $\mathcal{M}$ as a dense closed subset. The so-called Reid's fantasy suggests that an analogous situation could happen for Calabi-Yau threefolds where birational transformations and g.t.'s could be the right instruments to reduce the parameterization of birational classes of Calabi-Yau threefolds to an irreducible moduli space of complex structures over suitable connected sums of complex hypertori.

In physics, Calabi-Yau threefolds made their appearance in the eighties [5], as the space spanned by the so-called internal degrees of freedom of a certain $(1+1)-$ dimensional world-sheet field theory describing superstrings' propagation in $(3+1)-$ dimensional Minkowski space-time. The main observables of the superstring model are then typically determined by this internal structure (the Calabi-Yau vacuum). Therefore, in spite of the almost uniqueness, via dualities (and, later, $\mathcal{M}$-theory), of consistent superstring theories, the superstring model remains undetermined due to the unavoidable uncertainty on the topology of the Calabi-Yau vacuum: this is the so called vacuum degeneracy problem. A solution to this problem was firstly proposed by P.S. Green and T. Hübsch [12, 13, who conjectured, motivated by the contemporary Reid's fantasy, that topologically distinct Calabi-Yau vacua could be connected to each other by means of conifold transitions (see Definition 1.5) which should induce a phase transition between corresponding superstring models. This latter fact, which is the physical counterpart of the mathematical process given by a conifold transition, was actually understood by A. Strominger as a condensation of massive black holes to massless ones [49].

In this context it seems, then, of primary importance to understand and quantify the change in topology induced by a geometric transition. This is what $\mathrm{H}$. Clemens did for conifold transitions some time ago in [6]. For more general g.t's, a lot of computations have been carried out by many authors, in the last thirty years, but I wasn't able to find in the literature a clear and/or complete statement about these. Moreover, many results are obtained by invoking arguments which are not strictly topological, like, e.g., complex moduli and the Bogomolov-Tian-Todorov Theorem.

In the present paper I will try to organize this problem by introducing the concept of the homological type of a geometric transition. Precisely,

Definition 1. A g.t. $T=T(Y, \bar{Y}, \widetilde{Y})$ is said to admit homological type

$$
h[T]=\left(k^{\prime}, k^{\prime \prime}, c^{\prime}, c^{\prime \prime}\right)
$$


where $k^{\prime}, k^{\prime \prime}$ are non-negative integers and $c^{\prime}, c^{\prime \prime} \in \mathbb{Z}$ with $c^{\prime} \equiv c^{\prime \prime} \bmod 2$, if $T$ induces the following change on

(a) Betti numbers: $b_{i}(Y)=b_{i}(\bar{Y})=b_{i}(\widetilde{Y})$ for $i \neq 2,3,4$ and

$$
\begin{aligned}
& b_{2}(Y)=b_{2}(\bar{Y})+k^{\prime}+k^{\prime \prime}=b_{2}(\widetilde{Y})+k^{\prime}+k^{\prime \prime} \text {, } \\
& b_{3}(Y)=b_{3}(\bar{Y})-c^{\prime}=b_{3}(\tilde{Y})-c^{\prime}-c^{\prime \prime}, \\
& b_{4}(Y)=b_{4}(\bar{Y})+k^{\prime \prime}=b_{4}(\widetilde{Y})+k^{\prime}+k^{\prime \prime} .
\end{aligned}
$$

Then in particular $T$ induces the following changes on

(b) Hodge numbers:

$$
\begin{aligned}
& h^{1,1}(Y)=h^{1,1}(\widetilde{Y})+k \\
& h^{2,1}(Y)=h^{2,1}(\widetilde{Y})-c
\end{aligned}
$$

where $k:=k^{\prime}+k^{\prime \prime}$ and $c:=\left(c^{\prime}+c^{\prime \prime}\right) / 2$,

(c) Euler characteristics:

$$
\left.\begin{array}{c}
\chi(Y)-\chi(\bar{Y})=k+c^{\prime}+k^{\prime \prime} \\
\chi(\bar{Y})-\chi(\widetilde{Y})=k^{\prime}+c^{\prime \prime}
\end{array}\right\} \Rightarrow \chi(Y)-\chi(\widetilde{Y})=2(k+c) .
$$

Moreover, it turns out that $k^{\prime}=0$ if and only if $\bar{Y}$ is $\mathbb{Q}$-factorial (see Remark 4.4 and Lemma 5.4).

This notation is motivated by equations (b) on Hodge numbers allowing us to conclude that a g.t. $T(Y, \bar{Y}, \widetilde{Y})$ admitting homological type $h(T)=\left(k^{\prime}, k^{\prime \prime}, c^{\prime}, c^{\prime \prime}\right)$ increases complex moduli (in physics: hypermultiplets) by $c$ and decreases Kälher moduli (in physics: vector multiplets) by $k$, when passing from the Calabi-Yau 3-fold $Y$ to the Calabi-Yau 3-fold $\widetilde{Y}$.

Main Results. Let us first of all observe that a general g.t. $T(Y, \bar{Y}, \tilde{Y})$ does not admit a homological type since the homology change induced by $T$ cannot be summarized by a string of 4 integers: in fact in general $b_{2}(\bar{Y}) \neq b_{2}(\tilde{Y})$ requires the introduction of at least one further integer gauging this last discrepancy. By the way, quantifying the topological change given by a general g.t. is actually a hopeless problem since the exceptional locus $E:=\operatorname{Exc}(\phi)$ of the associated birational contraction $\phi: Y \rightarrow \bar{Y}$ may be very wild! On the other, hand what is observed in the present paper is that a homological type as in Definition 1 may suffice to describe what happens for some classes of (understandable) g.t's. Precisely, it is proven that:

Theorem 4.3 A small g.t. $T(Y, \bar{Y}, \tilde{Y})$ (see Definition 1.11) admits homological type

$$
h[T]=\left(k, 0, c^{\prime}, c^{\prime \prime}\right)
$$

where $k$ is the number of homologically independent exceptional rational curves composing $E=\operatorname{Exc}(\phi) \subset Y, c^{\prime}$ is the number of independent relations linking the homology classes of exceptional rational curves in $Y$ and $c^{\prime \prime}$ is the number of homologically independent vanishing cycles in $\widetilde{Y}$. Moreover,

(i) the total number of irreducible components of $E$ is

$$
n:=\sum_{p \in \operatorname{Sing}(\bar{Y})} n_{p}=k+c^{\prime}
$$


where $n_{p}$ is the number of irreducible (rational) components of $E_{p}:=\phi^{-1}(p)$, for any $p \in \operatorname{Sing}(\bar{Y})$,

(ii) the global Milnor number of $\bar{Y}$ is

$$
m:=\sum_{p \in \operatorname{Sing}(\bar{Y})} m_{p}=k+c^{\prime \prime},
$$

where $m_{p}$ is the Milnor number of the singular point $p \in \operatorname{Sing}(\bar{Y})$. Hence, $k$ turns out to be also the maximal number of independent relations linking the homology classes of vanishing cycles in $\widetilde{Y}$.

In particular, $T$ is a type $I$ g.t. (see Definition 1.10) if and only if $k^{\prime}=1=k$ and if $T$ is a conifold $t$., then $c^{\prime}=c^{\prime \prime}=c$.

Theorem 5.2 A type II g.t. $T(Y, \bar{Y}, \tilde{Y})$ (see Definition 1.10) admits homological type

$$
h[T]=\left(0,1, c^{\prime}, c^{\prime \prime}\right)
$$

given by

(i) $c^{\prime}=-1+b_{2}(E)-b_{3}(E)$,

(ii) $c^{\prime \prime}=1-\chi(\widetilde{B})=m_{p}-b_{2}(\widetilde{B})$,

where $\widetilde{B}$ is the Milnor fiber of the smoothing $\widetilde{Y}$ and $m_{p}:=b_{3}(\widetilde{B})$ is the Milnor number of the unique singular point $p=\phi(E) \in \bar{Y}$. In particular $b_{1}(\widetilde{B})=0$.

Let us say a few words about the previous results.

When $T$ is a conifold t., Theorem 4.3 gives precisely the Clemens' results in 6, here reported in Theorem 3.1. Moreover, relations (ii) and part of relations in Definition 1.(a), with $k^{\prime}$ and $c^{\prime \prime}$ as given in the statement of Theorem 4.3, were already proved by Y. Namikawa and J.H.M. Steenbrink in [36] Theorem (3.2) and Example (3.8): as far as I know, these were the most complete known evaluations of homology change induced by a small g.t. until now.

Theorem 5.2 can be improved by specializing the assumptions. In fact, it is well known that the exceptional locus $E=\operatorname{Exc}(\phi)$ of the birational contraction $Y \stackrel{\phi}{\rightarrow} \bar{Y}$, associated with a type II g.t. $T(Y, \bar{Y}, \widetilde{Y})$, is an irreducible generalized del Pezzo surface (39, Proposition (2.13)). This means that one of the following happens

- $E$ is normal and rational then Theorem 5.2 can be improved as follows (see Theorem 5.3):

(i) $c^{\prime}=9-d-n_{E}$, where $d=\operatorname{deg}(E):=\omega_{E}^{2}$ and $n_{E}$ is the number of rational exceptional curves composing the exceptional locus of a minimal resolution $\widehat{E} \stackrel{\pi}{\rightarrow} E$,

(ii) if $d \leq 4$ then $c^{\prime \prime}=m_{p}$.

- E admits an elliptic singular point (in the sense of [39], Definition (2.4)) then Theorem 5.2 can be improved as follows (see Theorem 5.7):

(i) $b_{2}(E)=1$ and $b_{3}(E)=2$, giving $c^{\prime}=-2$,

(ii) $c^{\prime \prime}=m_{p}$ and in particular it must be even.

- E is a non-normal del Pezzo surface (in the sense of [42]) then Theorem 5.2 can be improved as follows (see Theorem 5.13):

(i) $b_{2}(E) \in\{1,2\}$ and $b_{3}(E)=0$, giving $c^{\prime} \in\{0,1\}$, respectively,

(ii) $\chi(\widetilde{B}) \equiv b_{2}(E) \bmod 2$. 
The first equation in Definition 1.(b) in the case of a type II g.t., hence giving $k=1$, was already proved in 22] Proposition 3.1. In particular, if $E=\operatorname{Exc}(\phi)$ is smooth then a comparison between the equations in Definition 1.(b) and Theorem 3.3 and Remark 3.6 in [22], gives conditions on the Euler characteristic $\chi(\widetilde{B})$ and the Milnor number $m_{p}$ and then the list in Remark 5.6. In particular, this shows that we cannot expect the Milnor fiber near $p \in \bar{Y}$ to be a bouquet of 3-dimensional spheres when $d:=\operatorname{deg}(E)=6$ or 7 , the contrary of what happens for $d \leq 4$. Moreover, a further comparison with $[33 \quad \S 7.1$ and $[32$ \$3 gives an interesting interpretation of the homological type of a type II g.t. in terms of (dual) Coxeter numbers of suitable Weyl groups, as in (59).

Employed techniques are almost completely topological: construction of strong deformation retractions, Mayer-Vietoris and relative homology long exact sequences, dualities in homology, Leray spectral sequences and associated lower terms exact sequences. Transcendental methods (exponential sequence on a Calabi-Yau 3-fold and its pushed forward by birational contraction on a normal and singular 3-fold) are used simply to focus on how the Picard group is actually a topological invariant of all the 3 -folds involved in a g.t. .

The present paper is organized as follows. $\S 1$ reviews what a g.t. is, the related nomenclature, and Wilson's classification of birational contractions of Calabi-Yau 3 -folds. In $\S 2$ Milnor's and Looijenga's local analysis of isolated singularities are reviewed and the strong deformation retractions useful in the following are introduced (Propositions 2.4 and 2.7). In $\S 3$ we review a result proved in [43], rewritten in terms of the homological type of conifold transitions. Finally $\S 4$ and $\S 5$ state and prove the main results, the former for small g.t's and the latter for type II g.t's .

Acknowledgments. I would like to thank Alberto Collino for useful suggestions.

\section{Geometric transitions}

Definition 1.1 (Calabi-Yau 3-folds). A smooth, complex, projective 3-fold $Y$ is called Calabi-Yau if

(a) $\mathcal{K}_{Y} \cong \mathcal{O}_{Y}$,

(b) $h^{1,0}(Y)=h^{2,0}(Y)=0$.

Remark 1.2. There are a lot of more or less equivalent definitions of Calabi-Yau 3folds e.g.: a Kähler complex, compact 3-fold admitting either (1) a Ricci flat metric (Calabi conjecture and Yau theorem), or (2) a flat, non-degenerate, holomorphic 3form, or (3) holonomy group a subgroup of SU(3) (see 21] for a complete description of equivalences and implications).

In the algebraic context, the given definition gives the 3-dimensional analogous of smooth elliptic curves and smooth $K 3$ surfaces.

Examples 1.3. (a) Smooth hypersurfaces of degree 5 in $\mathbb{P}^{4}$,

(b) the general element of the anti-canonical system of a sufficiently good 4dimensional toric Fano variety (see [4]),

(c) suitable complete intersections.... (iterate the previous examples),

(d) the double covering of $\mathbb{P}^{3}$ ramified along a smooth surface of degree 8 in $\mathbb{P}^{3}$ (octic double solid). 
Definition 1.4 (Geometric transitions). (cfr. 31, 7], 11, [43]) Let $Y$ be a Calabi-Yau 3-fold and $\phi: Y \rightarrow \bar{Y}$ be a birational contraction onto a normal variety. If there exists a complex deformation (smoothing) of $\bar{Y}$ to a Calabi-Yau 3-fold $\tilde{Y}$, then the process of going from $Y$ to $\widetilde{Y}$ is called a geometric transition (for short transition or g.t.) and denoted by $T(Y, \bar{Y}, \widetilde{Y})$ or by the diagram

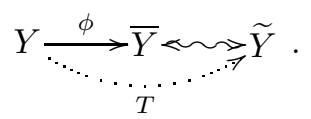

A transition $T(Y, \bar{Y}, \widetilde{Y})$ is called trivial if $\widetilde{Y}$ is a deformation of $Y$.

Definition 1.5 (Conifold transitions). A g.t.

$$
Y \underset{\ddots \cdots \ldots \ldots \ldots \cdot r}{\stackrel{\phi}{\longrightarrow}} \widetilde{Y}
$$

is called conifold (c.g.t. for short) and denoted $C T(Y, \bar{Y}, \widetilde{Y})$, if $\bar{Y}$ admits only ordinary double points (nodes or o.d.p.) as singularities.

Example 1.6 (cfr. 14]). The following is a non-trivial c.g.t.. For details see [43, 1.3 .

Let $\bar{Y} \subset \mathbb{P}^{4}$ be the generic quintic 3-fold containing the plane $\pi: x_{3}=x_{4}=0$. Its equation is

$$
x_{3} g\left(x_{0}, \ldots, x_{4}\right)+x_{4} h\left(x_{0}, \ldots, x_{4}\right)=0
$$

where $g$ and $h$ are generic homogeneous polynomials of degree $4 . \bar{Y}$ is then singular and

$$
\operatorname{Sing}(\bar{Y})=\left\{[x] \in \mathbb{P}^{4} \mid x_{3}=x_{4}=g(x)=h(x)=0\right\}=\{16 \text { nodes }\} .
$$

Blow up $\mathbb{P}^{4}$ along the plane $\pi$ and consider the proper transform $Y$ of $\bar{Y}$. Then:

- $Y$ is a smooth, Calabi-Yau 3-fold,

- the restriction to $Y$ of the blow up morphism gives a crepant resolution $\phi: Y \rightarrow \bar{Y}$.

The obvious smoothing of $\bar{Y}$ given by the generic quintic 3 -fold $\tilde{Y}$ completes the c.g.t. $C T(Y, \bar{Y}, \tilde{Y})$.

Definition 1.7 (Primitive contractions and transitions). A birational contraction from a Calabi-Yau 3-fold to a normal one is called primitive if it cannot be factored into birational morphisms of normal varieties. A g.t.

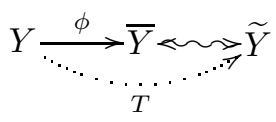

is called primitive if the associated birational contraction $\phi$ is primitive.

Proposition 1.8. Let $T(Y, \bar{Y}, \widetilde{Y})$ be a g.t. and $\phi: Y \rightarrow \bar{Y}$ the associated birational contraction. Then $\phi$ can always be factored into a composite of a finite number of primitive contractions.

Proof. The statement follows from the fact that any primitive contraction reduces by 1 the Picard number $\rho=\operatorname{rk}(\operatorname{Pic}(Y))=h^{1,1}(Y)$.

Theorem 1.9 (Classification of primitive contraction [54, [55]). Let $\phi: Y \rightarrow \bar{Y}$ be a primitive contraction from a Calabi-Yau threefold to a normal variety and let $E$ be the exceptional locus of $\phi$. Then one of the following is true: 
type I: $\phi$ is small which means that $E$ is composed of finitely many rational curves;

type II: $\phi$ contracts a divisor down to a point; in this case $E$ is irreducible and in particular it is a (generalized) del Pezzo surface (see [39])

type III: $\phi$ contracts a divisor down to a curve $C$; in this case $E$ is still irreducible and it is a conic bundle over a smooth curve $C$.

Definition 1.10 (Classification of primitive transitions). A transition $T(Y, \bar{Y}, \widetilde{Y})$ is called of type I, II or III if it is primitive and if the associated birational contraction $\phi: Y \rightarrow \bar{Y}$ is of type I, II or III, respectively.

Definition 1.11 (Small geometric transition). A transition

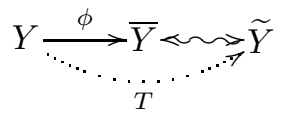

will be called small if $\phi$ is the composition of primitive contractions of type I.

\section{LOCAL ANALYSIS OF AN ISOLATED SINGULARITY}

Let $p \in \bar{Y}$ be a $n$-dimensional isolated singularity i.e. there exists a $n$-dimensional local analytic neighborhood $\bar{U}$ of $p$ bi-holomorphic to the zero locus of a polynomial map

$$
\mathbf{f}=\left(f_{1}, \ldots, f_{M}\right): \mathbb{C}^{N} \rightarrow \mathbb{C}^{M}
$$

where $N-M \leq n$ and such that $\mathbf{f}$ has an isolated critical point in $0 \in \mathbb{C}^{N}$ and $\mathbf{f}$ is a submersion over $\mathbb{C}^{N} \backslash\{0\}$. If $N-M=n$ then $p \in \bar{Y}$ is an isolated complete intersection singularity (i.c.i.s.). Moreover, if $M=1$, then $N=n+1$, and $p \in \bar{Y}$ is an isolated hypersurface singularity (i.h.s). Let $D_{\varepsilon}$ denote the closed $\varepsilon$-ball centered in $0 \in \mathbb{C}^{N}$ whose boundary is the $(2 N-1)$-dimensional $\varepsilon$-sphere $S_{\varepsilon}$.

Definition 2.1 (Good representatives and Milnor fibers, 27] Section 2.B). Let $T^{m}$ be a $m$-dimensional contractible neighborhood of $0 \in \mathbb{C}^{M}$, with $m \leq M$, and consider $\mathcal{U}^{m}:=\mathbf{f}^{-1}\left(T^{m}\right)$. Then

$$
\mathbf{f}: \mathcal{U}^{m} \longrightarrow T^{m}
$$

is called a good representative of $p$. We will omit the dimension $m$ if unnecessary. Set $\widetilde{U}:=U_{t}$ for $t \in T \backslash\{0\}$. Then, for small $\varepsilon>0$, the intersection

$$
\widetilde{B}=\widetilde{U} \cap D_{\varepsilon}
$$

is called the Milnor fiber of $p$.

Definition 2.2. Let $X \subset \mathbb{C}^{N}$ be a subset. The following subset of $\mathbb{C}^{N}$

$$
\mathrm{Cn}_{0}^{\varepsilon}(X):=\{t x \mid \forall t \in[0, \varepsilon] \subset \mathbb{R}, \forall x \in X\}
$$

will be called the $\varepsilon$-cone projecting $X$. The 1 -cone will be simply called the cone projecting $X$ and denoted by $\mathrm{Cn}_{0}(X)$. Moreover, the $\infty$-cone $\mathrm{Cn}_{0}^{\infty}(X)$ will be also called the unbounded cone projecting $X$.

Theorem 2.3 (Local topology of an isolated singularity, 27] Proposition (2.4), 28. Theorem 2.10, Theorem 5.2). Since $p \in \bar{U}$ is the unique critical point of $\mathbf{f}$, for any $\varepsilon>0$ there exists a homeomorphism $\psi_{\varepsilon}$ between the intersection

$$
\bar{B}:=\bar{U} \cap D_{\varepsilon}
$$


and the $\varepsilon$-cone projecting $K:=\bar{U} \cap S_{\varepsilon}$. Moreover, $\frac{1}{\varepsilon} \psi_{\varepsilon}$ gives a homeomorphism $\bar{B} \cong \mathrm{Cn}_{0}(K)$.

$K$ is called the link of $p:$ it only depends on the abstract analytic germ $(\bar{Y}, p)$ (see [27, Corollary (2.6)).

Proposition 2.4. In the same notation as before, $\bar{B}$ and $\widetilde{B}$ are strong deformation retract of $\bar{U}$ and $\widetilde{U}$, respectively.

Proof. Let us first of all observe that, by direct limit construction, there exists an homoeomorphism

$$
\varphi: \bar{U} \stackrel{\cong}{\longrightarrow} \mathrm{Cn}_{0}^{\infty}(K)
$$

where $K:=\bar{U} \cap S_{\varepsilon}$ for a sufficiently small $\varepsilon>0$. In fact, for any $0<\varepsilon^{\prime}<\varepsilon^{\prime \prime}$, Theorem 2.3 gives the following commutative diagram

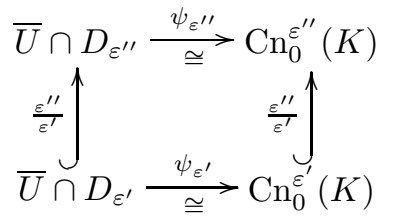

which, passing to direct limits $\bar{U}$ and $\mathrm{Cn}_{0}^{\infty}(K)$, gives the claimed homeomorphism $\varphi$. Moreover, by setting $\psi:=\frac{1}{\varepsilon} \psi_{\varepsilon}$, we get a homeomorphism

$$
\psi: \bar{B} \stackrel{\cong}{\longrightarrow} \mathrm{Cn}_{0}(K) \text {. }
$$

Let us start by showing the strong retraction $\bar{U} \approx \bar{B}$ : calling $i: \bar{B} \hookrightarrow \bar{U}$ the natural inclusion, we will write explicitly the retraction $\bar{r}: \bar{U} \rightarrow \bar{B}$ and the homotopy $\bar{F}: \bar{U} \times[0,1] \rightarrow \bar{U}$ such that $\operatorname{id}_{\bar{U}} \sim_{\bar{F}} i \circ \bar{r}$, since they will be useful in the following. Let

$$
r: \mathrm{Cn}_{0}^{\infty}(K) \longrightarrow \mathrm{Cn}_{0}(K)
$$

the obvious retraction defined by setting $r(x):=\left\{\begin{array}{cc}x & \text { if } x \in \mathrm{Cn}_{0}(K) \\ x /|x| & \text { otherwise }\end{array}\right.$. Consider the straight line homotopy

$$
H: \mathrm{Cn}_{0}^{\infty}(K) \times[0,1] \longrightarrow \mathrm{Cn}_{0}^{\infty}(K)
$$

defined by setting $H(x, t):=\left\{\begin{array}{cc}x & \text { if } x \in \mathrm{Cn}_{0}(K) \\ (1-t) x+t x /|x| & \text { otherwise }\end{array}\right.$.

$H$ is clearly continuous by the gluing lemma over $K$ whose points have unitary modulus. Moreover:

- $H(x, 0)=\operatorname{id}_{\mathrm{Cn}_{0}^{\infty}(K)}(x)=x$ and $H(x, 1)=r(x)$, which means that $\mathrm{Cn}_{0}(K)$ is a deformation retract of $\mathrm{Cn}_{0}^{\infty}(K)$,

- $H(x, t)=x$ for every $x \in \mathrm{Cn}_{0}(K)$, which means that $\mathrm{Cn}_{0}(K)$ is a strong deformation retract of $\mathrm{Cn}_{0}^{\infty}(K)$.

We are now able to construct the retraction $\bar{r}$ and the homotopy $\bar{F}$ as follows

$$
\begin{aligned}
\bar{r} & :=\psi^{-1} \circ r \circ \varphi \\
\bar{F}(z, t) & :=\varphi^{-1}(H(\varphi(z), t)) \quad, \quad \forall(z, t) \in \bar{U} \times[0,1]
\end{aligned}
$$

(notice that $\psi=\varphi \circ i \Rightarrow i \circ \psi^{-1}=\left.\varphi^{-1}\right|_{\mathrm{Cn}_{0}(K)}$ ). 
To prove the strong retraction $\widetilde{U} \approx \widetilde{B}$ set $\mathcal{B}_{\varepsilon}:=\mathcal{U}_{\varepsilon} \cap D_{\varepsilon}, \mathcal{K}_{\varepsilon}:=\mathcal{U}_{\varepsilon} \cap S_{\varepsilon}$ and consider the restricted fibration

$$
f_{\llcorner}: \mathcal{U}_{\varepsilon} \backslash \mathcal{B}_{\varepsilon} \cup \mathcal{K}_{\varepsilon} \longrightarrow T \text {. }
$$

Up to shrink $T$, the map $f_{\llcorner}$gives a fibration in $2 n$-dimensional $\mathcal{C}^{\infty}$-manifolds with boundary whose fibers are diffeomorphic to each other by the Ehresmann fibration theorem. Let then

$$
\eta: \widetilde{U} \backslash \widetilde{B} \cup \widetilde{K} \stackrel{\cong}{\longrightarrow} \backslash \bar{B} \cup \bar{K}
$$

be the induced diffeomorphism between the fibers $f_{\llcorner}^{-1}(t) \cong f_{\llcorner}^{-1}(0)$. We can then construct a retraction $\widetilde{r}: \widetilde{U} \rightarrow \widetilde{B}$ and a homotopy $\widetilde{F}: \widetilde{U} \times[0,1] \rightarrow \widetilde{U}$ as follows

$$
\begin{aligned}
\widetilde{r}(z) & :=\left\{\begin{array}{cc}
z & \text { if } z \in \widetilde{B} \\
\eta^{-1} \circ \bar{r} \circ \eta(z) & \text { otherwise }
\end{array}\right. \\
\widetilde{F}(z, t) & :=\left\{\begin{array}{cc}
z & \text { if } z \in \widetilde{B} \\
\eta^{-1}(\bar{F}(\eta(z), t)) & \text { otherwise }
\end{array}\right.
\end{aligned}
$$

Both $\widetilde{r}$ and $\widetilde{F}$ are continuous by the gluing lemma over $\widetilde{K}$ and the proof concludes immediately by verifying that $\operatorname{id}_{\widetilde{U}} \sim_{\widetilde{F}} \widetilde{i} \circ \widetilde{r}$, where $\widetilde{i}: \widetilde{B} \hookrightarrow \widetilde{U}$ is the inclusion.

Theorem 2.5 (Local homotopy type of the smoothing, 27] (5.6), (5.8) and Corollary (5.10), 28 Theorems 5.11, 6.5, 7.2). The Milnor fiber $\widetilde{B}$ is homotopy equivalent to a finite cell complex of dimension $\leq n$. Moreover, if $0 \in \mathbf{f}^{-1}(0)$ is an i.c.i.s. then $\widetilde{B}$ is $(n-1)$-connected and has the homotopy type of a finite bouquet of $n$-spheres.

In the following the $n$-th Betti number $b_{n}(\widetilde{B})$ of the Milnor fiber will be called the Milnor number of $p \in \bar{U} \subseteq \bar{Y}$ and denoted by $m_{p}$.

Remark 2.6. If $p \in \bar{U}$ is a $n$-dimensional i.h.s. then the Milnor number $m_{p}$ coincides with the multiplicity of $p$ as a critical point of the polynomial map $f$, which is the multiplicity of $0 \in \mathbb{C}^{n+1}$ as solution to the collection of polynomial equations

$$
\frac{\partial f}{\partial x_{1}}=\cdots=\frac{\partial f}{\partial x_{n+1}}=0 \text {. }
$$

The Milnor number is then given by

$$
m_{p}=\operatorname{dim}_{\mathbb{C}}\left(\mathbb{C}\left\{x_{1}, \ldots, x_{n+1}\right\} /\left(\frac{\partial f}{\partial x_{1}}, \cdots, \frac{\partial f}{\partial x_{n+1}}\right)\right)
$$

where $\mathbb{C}\left\{x_{1}, \ldots, x_{n+1}\right\}$ is the $\mathbb{C}$-algebra of converging power series.

Proposition 2.7 (Local topology of the resolution). Let $\phi: U \rightarrow \bar{U}$ be a birational resolution of the i.s. $p \in \bar{U}$ and set $E:=\operatorname{Exc}(\phi)$. The pull back by $\phi$ of the strong deformation retraction $\bar{U} \approx \bar{B} \approx\{p\}$ gives rise to a strong deformation retraction $U \approx B \approx E$, where $B:=\phi^{-1}(\bar{B})$.

Proof of Lemma 2.7. Consider the homeomorphisms $\varphi, \psi$ given by (11) and (2), respectively. Then the straight line homotopy giving the contraction $\bar{B} \approx\{p\}$ is the following

$$
\begin{aligned}
\bar{G}: \quad \bar{B} \times[0,1] & \longrightarrow \bar{B} \\
(z, t) & \longmapsto \psi^{-1}((1-t) \psi(z))
\end{aligned}
$$


Therefore the contraction $\bar{U} \approx\{p\}$ is realized by the composition $\bar{G} * \bar{F}$ where $\bar{F}$ is the homotopy defined in (3). Pulling back by the resolution $\phi: U \rightarrow \bar{U}$ we get retractions and homotopies

$$
\begin{array}{rll}
r & : & U \longrightarrow E \\
G * F & : \quad U \times[0,1] \longrightarrow U
\end{array}
$$

defined by setting

$$
\begin{aligned}
& r(z):=\left\{\begin{array}{cc}
z & \text { if } z \in E \\
\widehat{l}_{\phi(z)} \cap E & \text { otherwise }
\end{array}\right. \\
& G * F(z, t):=\left\{\begin{array}{cc}
z & \text { if } z \in E \\
\phi^{-1}(\bar{F}(\phi(z), 2 t)) & \text { if }(z, t) \in(U \backslash E) \times\left[0, \frac{1}{2}\right] \\
\phi^{-1}(\bar{G}(\bar{F}(\phi(z), 1), 2 t-1)) & \text { if }(z, t) \in(U \backslash E) \times\left[\frac{1}{2}, 1\right) \\
\widehat{l}_{\phi(z)} \cap E & \text { if }(z, t) \in(U \backslash E) \times\{1\}
\end{array}\right.
\end{aligned}
$$

where $\widehat{l}_{\phi(z)}$ is the strict transform by $\phi$ of the segment

$$
l_{\phi(z)}=\left\{\varphi^{-1}((1-t) \varphi(\phi(z))) \mid t \in[0,1]\right\}
$$

i.e. it is the closure of the subset $\left\{\Phi^{-1}((1-t) \Phi(z)) \mid t \in[0,1)\right\} \subset U$, where $\Phi:=\varphi \circ \phi$. Then $\widehat{l}_{\phi(z)} \cap E$ is its unique closure point. This gives the continuity of $r$ and of $G * F$ by the gluing lemma over $E$ and over $U \times\left\{\frac{1}{2}, 1\right\}$. Moreover,

- $G * F(z, 0)=z$ and $G * F(z, 1)=r(z)$, for every $z \in U$, which means that $E$ is a deformation retract of $U$,

- $G * F(z, t)=z$ for every $(z, t) \in E \times[0,1]$, which means that $E$ is a strong deformation retract of $U$.

\section{HomologicAl type OF A CONIFOlD tRANSITION}

Consider the following c.g.t.

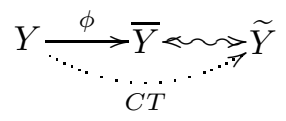

Then, by definition, $\operatorname{Sing}(\bar{Y})$ is entirely composed by a finite number of nodes. The following theorem can be obtained by summarizing several results of many authors. It gives a complete account of changing in topology induced by a c.g.t.

Theorem 3.1 (Global changing in topology for a conifold transition 6], 41], [52, [51, 36], 32, ...). Consider the c.g.t. [4]). Then

$$
h[C T]=(k, 0, c, c)
$$

where

- $k$ is the number of homologically independent exceptional $\mathbb{P}^{1} s$ in $Y$,

- $c$ is the number of homologically independent vanishing cycles in $\tilde{Y}$. Moreover, if $N:=\operatorname{Sing}(\bar{Y})$ is the number of nodes in $\bar{Y}$, then $N=k+c$.

A detailed topological proof of this theorem, based on the local Clemens' analysis described in [6], is given in [43], section 3 . 
Remark 3.2. Note that point (a) in Definition 1, with $k$ and $c$ as in Theorem 3.1. implies that the conifold $\bar{Y}$ do not satisfy Poincaré Duality. The difference $b_{4}(\bar{Y})-b_{2}(\bar{Y})=k$ is called the defect of $\bar{Y}$ [36]. See also the following remark 4.4. This fact can be deeply understood by means of homology of intersections spaces and intersection homology, as recently introduced and explained by M. Banagl in [2] $\S 5$, giving a nice account of relations with type II string theories and mirror symmetry in physics (see also the following remark 3.3).

Remark 3.3. Point (b) in Definition 1, with $k$ and $c$ as in Theorem 3.1, admits the following geometric (and physical) interpretation: a c.g.t. increases complex moduli (in phisics: hypermultiplets) by the number c of homologically independent vanishing cycles and decreases Kähler moduli (in phisics: vector multiplets) by the number $k$ of homologically independent exceptional rational curves.

Remark 3.4 (Example 1.6 continued). If Theorem 3.1 is applied to the c.g.t. in Example 1.6 one finds that $k^{\prime}=k=1, k^{\prime \prime}=0$, since $Y \stackrel{\phi}{\longrightarrow} \bar{Y}$ is primitive, as induced by a blow up. Then $c=N-k=16-1=15=c^{\prime}=c^{\prime \prime}$ and recalling that $\widetilde{Y}$ is a generic quintic 3 -fold in $\mathbb{P}^{4}$ one gets the following table of Betti numbers:

\begin{tabular}{c|ccc}
\hline \hline & $b_{2}$ & $b_{3}$ & $b_{4}$ \\
\hline & & & \\
$Y$ & 2 & 174 & 2 \\
$\bar{Y}$ & 1 & 189 & 2 \\
$\widetilde{Y}$ & 1 & 204 & 1 \\
\hline \hline
\end{tabular}

Then $\bar{Y}$ has defect 1, as observed in Remark 3.2

\section{Homological type of A SMALL GEOMETRIC TRANSITION}

Let

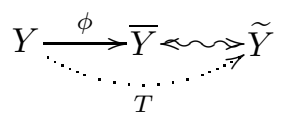

be a small g.t.. Then $\operatorname{Sing}(\bar{Y})$ is composed of a finite number of canonical singularities (see [39], section 1, for the definition) since $\operatorname{dim} \phi^{-1}(p)=1$, for any singular point $p \in \bar{Y}$.

Recall that a compound Du Val (cDV) singularity is a 3 -fold point $p$ such that, for a hyperplane section $H$ through $p, p \in H$ is a Du Val surface singularity i.e. an A-D-E singular point (see 39, sections 0 and 2, and 3, chapter III). The singular $\operatorname{locus} P:=\operatorname{Sing}(\bar{Y})$ and the exceptional locus $E:=\phi^{-1}(P)$ have then a well known geometry reviewed by the following statement.

Theorem 4.1 (40], 26], 38, [30], 10]). Given a small g.t. as in (6) then:

(a) any $p \in P$ is a $c D V$ singularity,

(b) for any $p \in P, E_{p}:=\phi^{-1}(p)$ is a connected union of rational curves meeting transversally, whose configuration is dually represented either by one of the 


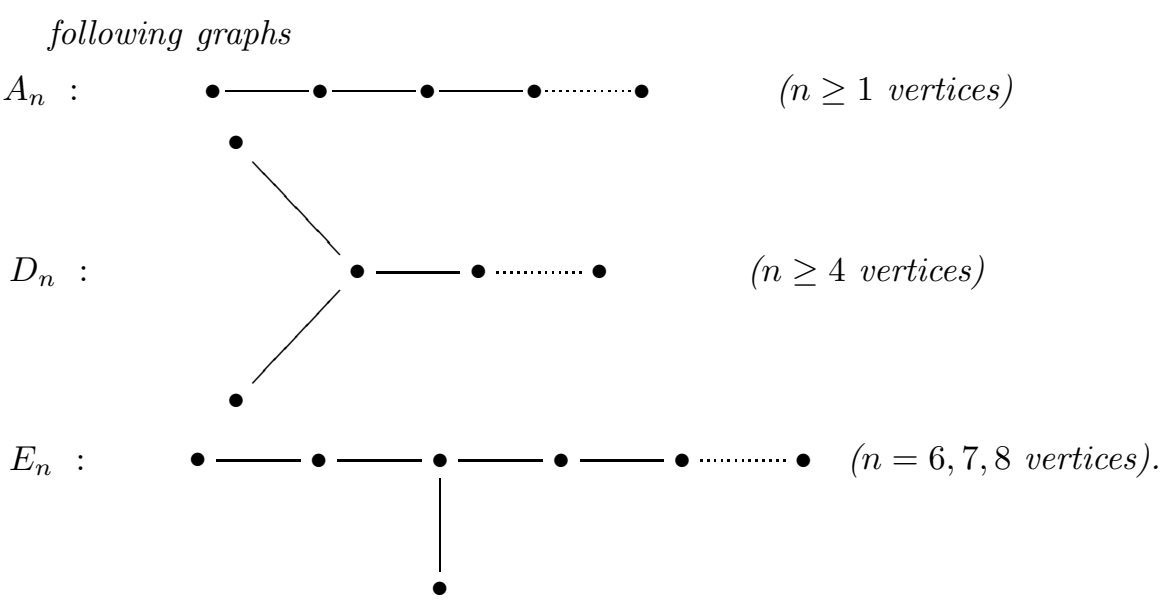

or, if $p$ is a non-planar singularity, by one the following graphs

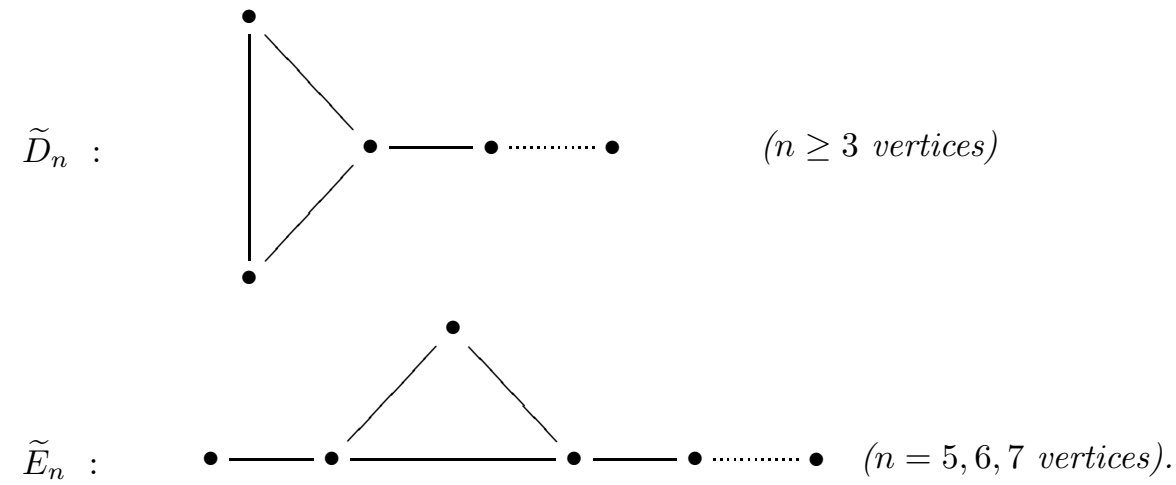

where triangles are dual graphs representing the transverse intersection of three rational curves at a single point.

Clearly every conifold transition is a small g.t. admitting exceptional trees of $A_{1}$ type. The following example presents a small non-conifold transition.

Example 4.2 (A small non-conifold g.t., 35] Example 1.11 and Remark 2.8, 44]). Let $S$ be the rational elliptic surface with sections obtained as the Weierstrass fibration associated with the bundles homomorphism

$$
\begin{aligned}
\beta: \mathcal{E}=\mathcal{O}_{\mathbb{P}^{1}}(3) \oplus \mathcal{O}_{\mathbb{P}^{1}}(2) \oplus \mathcal{O}_{\mathbb{P}^{1}} & \longrightarrow \mathcal{O}_{\mathbb{P}^{1}}(6) \\
(x, y, z) & \longmapsto-x^{2} z+y^{3}+B(\lambda) z^{3}
\end{aligned}
$$

for a generic $B \in H^{0}\left(\mathbb{P}^{1}, \mathcal{O}_{\mathbb{P}^{1}}(6)\right)$ i.e. $S$ is the zero locus of $\beta$ in the projectivized bundle $\mathbb{P}(\mathcal{E})$. Then:

- the natural fibration $S \rightarrow \mathbb{P}^{1}$ has generic smooth fiber and 6 distinct (since $B$ is generic) cuspidal fibers,

- the fiber product $\bar{Y}:=S \times_{\mathbb{P} 1} S$ is a threefold admitting 6 distinct singularities of type II $\times I I$, in the standard Kodaira notation [24], whose local equation in $\mathbb{C}^{4}$ is given by

$$
x^{2}-u^{2}=y^{3}-v^{3},
$$


- $\bar{Y}$ is a special fiber of the family of fiber products $S_{1} \times_{\mathbb{P}^{1}} S_{2}$ of rational elliptic surfaces with sections [46]: in particular every rational elliptic surface can be thought as the blow up of $\mathbb{P}^{2}$ at the base locus of a bi-cubic rational map $\mathbb{P}^{2} \stackrel{[a: b]}{\rightarrow} \mathbb{P}^{1}$ (34] Prop. 6.1) implying that $\bar{Y}$ can be thought as a special fiber of the family of smooth resolutions of bi-cubic hypersurfaces (44] Remark $2.2)$

$$
\left\{a(x) b^{\prime}\left(x^{\prime}\right)=a^{\prime}\left(x^{\prime}\right) b(x)\right\} \subset \mathbb{P}^{2}[x] \times \mathbb{P}^{2}\left[x^{\prime}\right], \quad a, b, a^{\prime}, b^{\prime} \in H^{0}\left(\mathcal{O}_{\mathbb{P}^{2}}(3)\right),
$$

- $\bar{Y}$ admits a small resolution $Y \stackrel{\phi}{\longrightarrow} \bar{Y}$ whose exceptional locus is composed by 6 disjoint couples of rational curves intersecting in one point i.e. 6 disjoint $A_{2}$ exceptional trees in the notation of Theorem 4.1](35] §0.1, 44] Proposition 3.1).

Let us say a few words about the construction of the resolution $Y \stackrel{\phi}{\longrightarrow} \bar{Y}$. The fibred product $\bar{Y}:=S \times_{\mathbb{P}^{1}} S$ can be thought as embedded in $\mathbb{P}:=\mathbb{P}(\mathcal{E}) \times \mathbb{P}(\mathcal{E}) \times \mathbb{P}^{1}$ as follows

$$
\mathbb{P}:=\mathbb{P}(\mathcal{E}) \times \mathbb{P}(\mathcal{E}) \times \mathbb{P}^{1}[\lambda] \supset \bar{Y}:\left\{\begin{array}{l}
x^{2} z=y^{3}+B(\lambda) z^{3} \\
u^{2} w=v^{3}+B(\lambda) z^{3}
\end{array} .\right.
$$

Consider the following cyclic map on $\mathbb{P}$

$$
\begin{aligned}
\tau: \quad & \mathbb{P}(\mathcal{E}) \times \mathbb{P}(\mathcal{E}) \times \mathbb{P}^{1} \longrightarrow \mathbb{P}(\mathcal{E}) \times \mathbb{P}(\mathcal{E}) \times \mathbb{P}^{1} \\
(x: y: z) & \longrightarrow(u: v: w) \times \lambda \longmapsto(x: y: z) \times(-u: \epsilon v: w) \times \lambda,
\end{aligned}
$$

where $\epsilon$ is a primitive cubic root of unity. The second equation in (10) ensures that $\tau Y=Y$. Moreover, for any $p \in \operatorname{Sing}(\bar{Y}), \tau(p)=p$, by (8). Consider the codimension 2 diagonal locus $\Delta:=\left\{\left(x, x^{\prime}, \lambda\right) \in \mathbb{P} \mid x=x^{\prime}\right\}$. Clearly $\operatorname{Sing}(\bar{Y}) \subset \Delta$, implying that $\operatorname{Sing}(\bar{Y})=\bar{Y} \cap \Delta \cap \tau \Delta$. Let then $Y$ be the strict transform of $\bar{Y}$ in the successive blow up

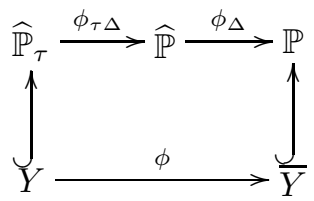

where $\phi_{\Delta}$ is the blow up of $\mathbb{P}$ along $\Delta$ and $\phi_{\tau \Delta}$ is the blow up of $\widehat{\mathbb{P}}$ along the strict transform $\widehat{\tau \Delta} \subset \widehat{\mathbb{P}}$ of $\tau \Delta$. Since $\phi$ turns out to be a small, crepant resolution, $Y$ is a Calabi-Yau threefold and

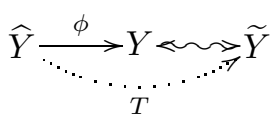

is a small non-conifold g.t. with 6 disjoint exceptional trees of type $A_{2}$, where $\tilde{Y}$ is the smooth small resolution of a bi-cubic hypersurface of type (9).

4.1. Homology change induced by a small geometric transition. We are now in a position to write down, for small g.t.'s, a statement analogous to Theorem 3.1. The following theorem is actually a revised version of results of Y. Namikawa and J.H.M. Steenbrink (see [36, Theorem (3.2) and Example (3.8)). After replacing Clemens' local analysis by Milnor's one, the proof given here is completely analogous to the proof of Theorem 3.1 given in [43]. 
Theorem 4.3. Consider the small g.t. (6). Then

$$
h[T]=\left(k, 0, c^{\prime}, c^{\prime \prime}\right)
$$

where

- $k$ is the number of homologically independent exceptional rational curves composing $E=\operatorname{Exc}(\phi) \subset Y$,

- $c^{\prime}$ is the number of independent relations linking the homology classes of exceptional rational curves in $Y$,

- $c^{\prime \prime}$ is the number of homologically independent vanishing cycles in $\tilde{Y}$.

Moreover,

(i) the total number of irreducible components of $E$ is

$$
n:=\sum_{p \in P} n_{p}=k+c^{\prime}
$$

where $n_{p}$ is the number of irreducible (rational) components of $E_{p}:=\phi^{-1}(p)$, for any $p \in P:=\operatorname{Sing}(\bar{Y})$,

(ii) the global Milnor number of $\bar{Y}$ is

$$
m:=\sum_{p \in P} m_{p}=k+c^{\prime \prime},
$$

where $m_{p}$ is the Milnor number of the singular point $p \in P$. Hence $k$ turns out to be also the number of independent relations linking the homology classes of vanishing cycles in $\tilde{Y}$.

In particular, $T$ is a type $I$ g.t. if and only if $k^{\prime}=1=k$ and if $T$ is a conifold $t$. then $c^{\prime}=c^{\prime \prime}=c$.

Remark 4.4. As in Remark 3.2, point (b) in Definition 1 with $k, c^{\prime}, c^{\prime \prime}$ as in Theorem 4.3, implies that $\bar{Y}$ has defect $k$.

On the other hand, by (a) of Theorem 4.1, any $p \in P$ is a rational i.h.s.. Moreover, $\bar{Y}$ is normal and $H^{2}\left(\bar{Y}, \mathcal{O}_{\bar{Y}}\right)=0$, since $Y$ is a Calabi-Yau 3-fold. Under all these conditions, Lemmas (3.3) and (3.5) in [36] apply to give that

$$
k=\operatorname{rk}\left(\langle\text { Weil divisors of } \bar{Y}\rangle_{\mathbb{Z}} /\langle\text { Cartan divisors of } \bar{Y}\rangle_{\mathbb{Z}}\right)
$$

Recall now that a variety is called $\mathbb{Q}$-factorial if any Weil divisor is a $\mathbb{Q}$-Cartier divisor. Then:

- given a small non trivial g.t. $T(Y, \bar{Y}, \tilde{Y})$, the singular 3-fold $\bar{Y}$ is never $\mathbb{Q}$-factorial.

In fact, any primitive extremal transition reduces by 1 the $\operatorname{rank}$ of $\operatorname{Pic}(Y) \cong$ $H^{2}(Y, \mathbb{Z})$. By Poinacaré duality on $Y$ the exceptional cycle of a small transition can never be homologically trivial.

Remark 4.5 (Example 4.2 continued). If Theorem 4.3 is applied to the small g.t. in Example 4.2, one finds that $k^{\prime}=k=2, k^{\prime \prime}=0$, since $Y \stackrel{\phi}{\longrightarrow} \bar{Y}$ is induced by two successive blow-ups. Then $c^{\prime}=n-k=12-2=10$. On the other hand the singular point (8) has Milnor number $m_{p}=4$, by the Milnor-Orlik Theorem ([29] Theorem 1). Then the global Milnor number of $\bar{Y}$ is given by $m=6 \cdot 4=24$ giving 
that $c^{\prime \prime}=m-k=24-2=22$. Therefore one gets the following table of Betti numbers (see [44, Theorem 3.8):

\begin{tabular}{l|lll}
\hline \hline & $b_{2}$ & $b_{3}$ & $b_{4}$ \\
\hline & & & \\
$Y$ & 21 & 8 & 21 \\
$\bar{Y}$ & 19 & 18 & 21 \\
$\widetilde{Y}$ & 19 & 40 & 19 \\
\hline \hline
\end{tabular}

Then $\bar{Y}$ has defect 2 .

Proof of Theorem 4.3. Given the small g.t. (6), for any $p \in P$ construct $\bar{U}_{p}, \bar{B}_{p}$ and $\widetilde{U}_{p}, \widetilde{B}_{p}$ like in Definition 2.1 and Theorem 2.3. Set

$$
U_{p}:=\phi^{-1}\left(\bar{U}_{p}\right) \quad, \quad B_{p}:=\phi^{-1}\left(\bar{B}_{p}\right) .
$$

We have then the following localization, near to $p$, of the small g.t. (6):

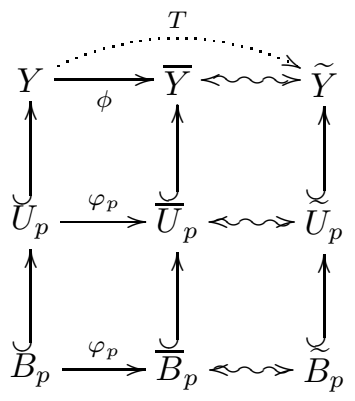

Let us denote:

$$
\begin{array}{lllll}
U:=\bigcup_{p \in P} U_{p} \quad, \quad B:=\bigcup_{p \in P} B_{p} \quad, \quad Y^{*}:=Y \backslash B \quad, \quad U^{*}:=U \backslash B ; \\
\bar{U}:=\bigcup_{p \in P} \bar{U}_{p} \quad, \quad \bar{B}:=\bigcup_{p \in P} \bar{B}_{p} \quad, \quad \bar{Y}^{*}:=\bar{Y} \backslash \bar{B} \quad, \quad \bar{U}^{*}:=\bar{U} \backslash \bar{B} ; \\
\widetilde{U}:=\bigcup_{p \in P} \widetilde{U}_{p} \quad, \quad \widetilde{B}:=\bigcup_{p \in P} \widetilde{B}_{p} \quad, \quad \widetilde{Y}^{*}:=\widetilde{Y} \backslash \widetilde{B}, & \widetilde{U}^{*}:=\widetilde{U} \backslash \widetilde{B} .
\end{array}
$$

We get then the following Mayer-Vietoris couples:

$$
\begin{aligned}
\mathcal{C} & :=\left(Y=Y^{*} \cup U, U^{*}=Y^{*} \cap U\right), \\
\overline{\mathcal{C}} & :=\left(\bar{Y}=\bar{Y}^{*} \cup \bar{U}, \bar{U}^{*}=\bar{Y}^{*} \cap \bar{U}\right), \\
\widetilde{\mathcal{C}} & :=\left(\widetilde{Y}=\widetilde{Y}^{*} \cup \widetilde{U}, \widetilde{U}^{*}=\widetilde{Y}^{*} \cap \widetilde{U}\right) .
\end{aligned}
$$

Step I. $\forall i \neq 2,3 \quad b_{i}(Y)=b_{i}(\bar{Y})$ and

$$
b_{2}(Y)-b_{2}(\bar{Y})=k \Leftrightarrow b_{3}(\bar{Y})-b_{3}(Y)=n-k .
$$

Compare the singular homology long exact sequences associated with $\mathcal{C}$ and $\overline{\mathcal{C}}$ :

$$
\cdots \longrightarrow H_{q}\left(U^{*}\right) \longrightarrow H_{q}\left(Y^{*}\right) \oplus H_{q}(U) \longrightarrow H_{q}(Y) \longrightarrow H_{q-1}\left(U^{*}\right) \longrightarrow \cdots
$$

$$
\cdots \longrightarrow H_{q}\left(\bar{U}^{*}\right) \longrightarrow H_{q}\left(\bar{Y}^{*}\right) \oplus H_{q}(\bar{U}) \longrightarrow H_{q}(\bar{Y}) \longrightarrow H_{q-1}\left(\bar{U}^{*}\right) \longrightarrow \cdots
$$


MICHELE ROSSI

Since $\phi$ is an isomorphism outside of the exceptional locus $E$ we get

$$
\begin{aligned}
\forall q \quad H_{q}\left(Y^{*}\right) & \cong H_{q}\left(\bar{Y}^{*}\right) \\
H_{q}\left(U^{*}\right) & \cong H_{q}\left(\bar{U}^{*}\right)
\end{aligned}
$$

Moreover, by proposition 2.4 $\bar{B}$ turns out to be a strong deformation retract of $\bar{U}$ and, by theorem $[2.3, \bar{B}$ is a union of cones which can be contracted, by straight line homotopy, to $P$. Then

$$
H_{q}(\bar{U}) \cong H_{q}(P) \cong\left\{\begin{array}{cc}
\mathbb{Z}^{|P|} & \text { if } q=0 \\
0 & \text { otherwise }
\end{array}\right.
$$

On the other hand, recalling Proposition 2.7 we get the following

$$
\forall q \quad H_{q}(U) \cong H_{q}(E)=\bigoplus_{p \in P} H_{q}\left(E_{p}\right) \cong \bigoplus_{p \in P} H_{q}\left(S^{2}\right)^{\oplus n_{p}} \cong\left\{\begin{array}{cl}
\mathbb{Z}^{|P|} & \text { for } q=0 \\
\mathbb{Z}^{n} & \text { for } q=2 \\
0 & \text { otherwise. }
\end{array}\right.
$$

In fact, let us proceed by induction. If $n_{p}=1$ then $E_{p} \cong S^{2}$. Point (b) in Theorem 4.1 allows us to think of $E_{p}$ as the union

$$
E_{p}=C \cup E^{\prime}
$$

where $C \cong \mathbb{P}_{\mathbb{C}}^{1}, E^{\prime}$ is a connected union of $n_{p}-1$ rational curves, whose configuration is still represented by one of the listed graphs, and $C \cap E^{\prime}$ is a single point $y$. Then $\left(E^{\prime}, C\right)$ gives a Mayer-Vietoris couple. Since

$$
H_{q}\left(C \cap E^{\prime}\right) \cong H_{q}(\{y\})=0 \quad \forall q \geq 1,
$$

the singular homology long exact sequence of $\left(E^{\prime}, C\right)$ allows us to conclude that

$$
H_{q}\left(E_{p}\right) \cong H_{q}(C) \oplus H_{q}\left(E^{\prime}\right) \quad \forall q \geq 2
$$

giving (18), for $q \geq 2$, by induction hypothesis. We have then the following exact sequence

$$
0 \rightarrow H_{1}(C) \oplus H_{1}\left(E^{\prime}\right) \rightarrow H_{1}\left(E_{p}\right) \rightarrow H_{0}(\{y\}) \rightarrow H_{0}(C) \oplus H_{0}\left(E^{\prime}\right) \rightarrow H_{0}\left(E_{p}\right) \rightarrow 0
$$

Since $\{y\}, C, E^{\prime}$ and $E_{p}$ are all connected, (18) follows for $q=1$, too.

Let us now conclude to prove Step I. By (14) and (15) and formula (17) we get that

$$
\forall q \neq 2,3 \quad b_{q}(Y)=b_{q}(\bar{Y})
$$

Moreover, the gluing of sequences (14) and (15), by identification of isomorphic poles, reduces to the following diagram

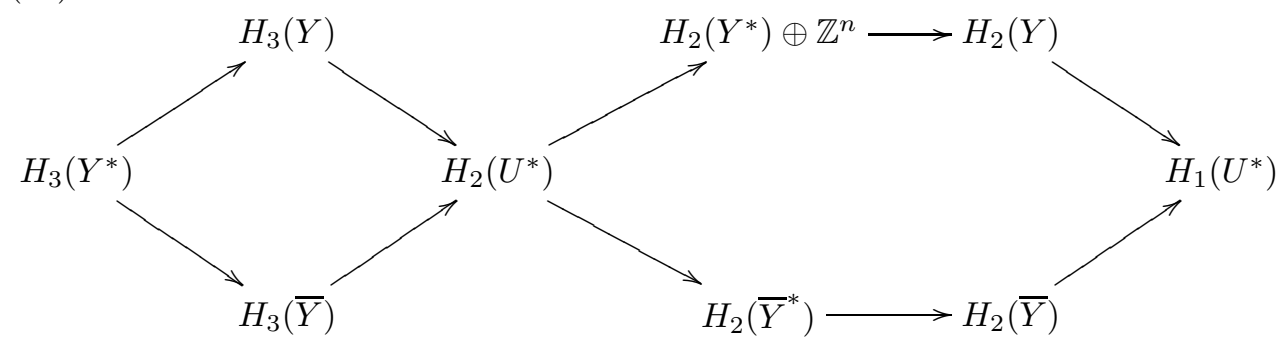

which gives the following relations between Betti numbers

$$
b_{2}(Y)-b_{2}(\bar{Y})=b_{3}(Y)-b_{3}(\bar{Y})+n \text {. }
$$


Step II. $\forall q \neq 3,4 \quad b_{q}(\tilde{Y})=b_{q}(\bar{Y})$ and

$$
b_{3}(\tilde{Y})-b_{3}(\bar{Y})=c^{\prime \prime} \Leftrightarrow b_{4}(\bar{Y})-b_{4}(\widetilde{Y})=m-c^{\prime \prime} .
$$

Compare the following singular homology long exact sequence associated with the couple $\widetilde{\mathcal{C}}$

$$
\cdots \longrightarrow H_{q}\left(\widetilde{U}^{*}\right) \longrightarrow H_{q}\left(\widetilde{Y}^{*}\right) \oplus H_{q}(\widetilde{U}) \longrightarrow H_{q}(\widetilde{Y}) \longrightarrow H_{q-1}\left(\widetilde{U}^{*}\right) \longrightarrow \cdots
$$

and the Mayer-Vietoris sequence (15) associated with $\overline{\mathcal{C}}$.

Recall that, by Proposition 2.4 $\widetilde{B}$ is a strong deformation retract of $\widetilde{U}$. Moreover, Theorem 2.5 asserts that, for any $p \in P$, the Milnor fiber $\widetilde{B}_{p}$ has the same homology type of a bouquet of $m_{p} 3$-dimensional spheres. Then

$$
H_{q}(\widetilde{U}) \cong H_{q}(\widetilde{B}) \cong \bigoplus_{p \in P} H_{q}\left(\widetilde{B}_{p}\right) \cong\left\{\begin{array}{cl}
\mathbb{Z}^{|P|} & \text { if } q=0 \\
\mathbb{Z}^{m} & \text { if } q=3 \\
0 & \text { otherwise. }
\end{array}\right.
$$

The localization (13) and the Ehresmann fibration theorem allow us to assert that there are diffeomorphisms

$$
\bar{Y}^{*} \cong \widetilde{Y}^{*} \text { and } \bar{U}^{*} \cong \widetilde{U}^{*}
$$

Then, recalling formulas (17) and (21), we can conclude that

$$
\forall q \neq 3,4 \quad b_{q}(\widetilde{Y})=b_{q}(\bar{Y}) .
$$

Moreover, the gluing of sequences (15) and (20), by identification of isomorphic poles, reduces to the following diagram

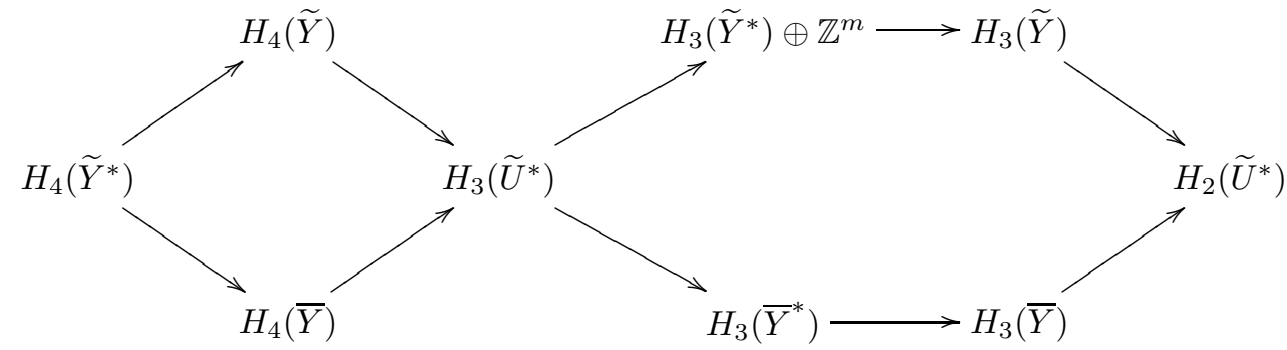

which gives the following relations between Betti numbers

$$
b_{3}(\tilde{Y})-b_{3}(\bar{Y})=b_{4}(\tilde{Y})-b_{4}(\bar{Y})+m .
$$

Step III. Let $k$ and $c^{\prime \prime}$ be the same parameters defined in Steps I and II respectively. Then

$$
m=k+c^{\prime \prime} .
$$

By Poincaré duality

$$
\begin{aligned}
& b_{2}(Y)=b_{4}(Y) \\
& b_{4}(\tilde{Y})=b_{2}(\widetilde{Y})
\end{aligned}
$$


Recall then Steps I and II to get

$$
\begin{aligned}
b_{2}(Y) & =b_{4}(Y)=b_{4}(\bar{Y})=b_{4}(\widetilde{Y})+m-c^{\prime \prime} \\
& =b_{2}(\widetilde{Y})+m-c^{\prime \prime}=b_{2}(\bar{Y})+m-c^{\prime \prime}=b_{2}(Y)-k+m-c^{\prime \prime}
\end{aligned}
$$

Then $m-k-c^{\prime \prime}=0$.

Step IV. $k$ is the maximal number of homologically independent exceptional rational curves in $Y$ while $c^{\prime \prime}$ is the maximal number of homologically independent vanishing cycles in $\widetilde{Y}$.

Since the birational contraction $\phi$ is an isomorphism outside of the exceptional locus $E$ and by the Ehresmann fibration theorem, we get the following composition of diffeomorphisms

$$
Y^{*} \cong \bar{Y}^{*} \cong \tilde{Y}^{*}
$$

and, by Lefschetz duality,

$$
H_{i}(Y, B) \cong H^{6-i}(Y \backslash B) \cong H^{6-i}(\widetilde{Y} \backslash \widetilde{B}) \cong H_{i}(\widetilde{Y}, \widetilde{B})
$$

Consider the long exact relative homology sequences of the couples $(Y, B)$ and $(\widetilde{Y}, \widetilde{B})$ and the vertical isomorphisms given by (23):

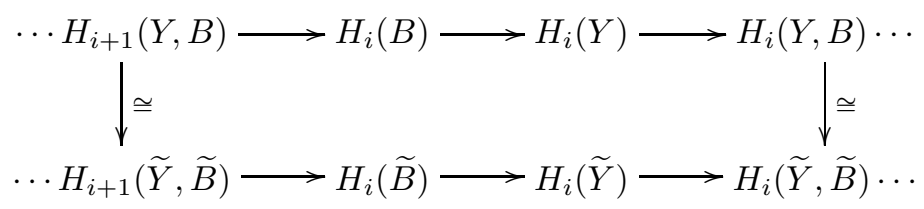

By identifying the isomorphic poles and recalling (18) and (21) the previous long exact sequences reduce to the following diagram:

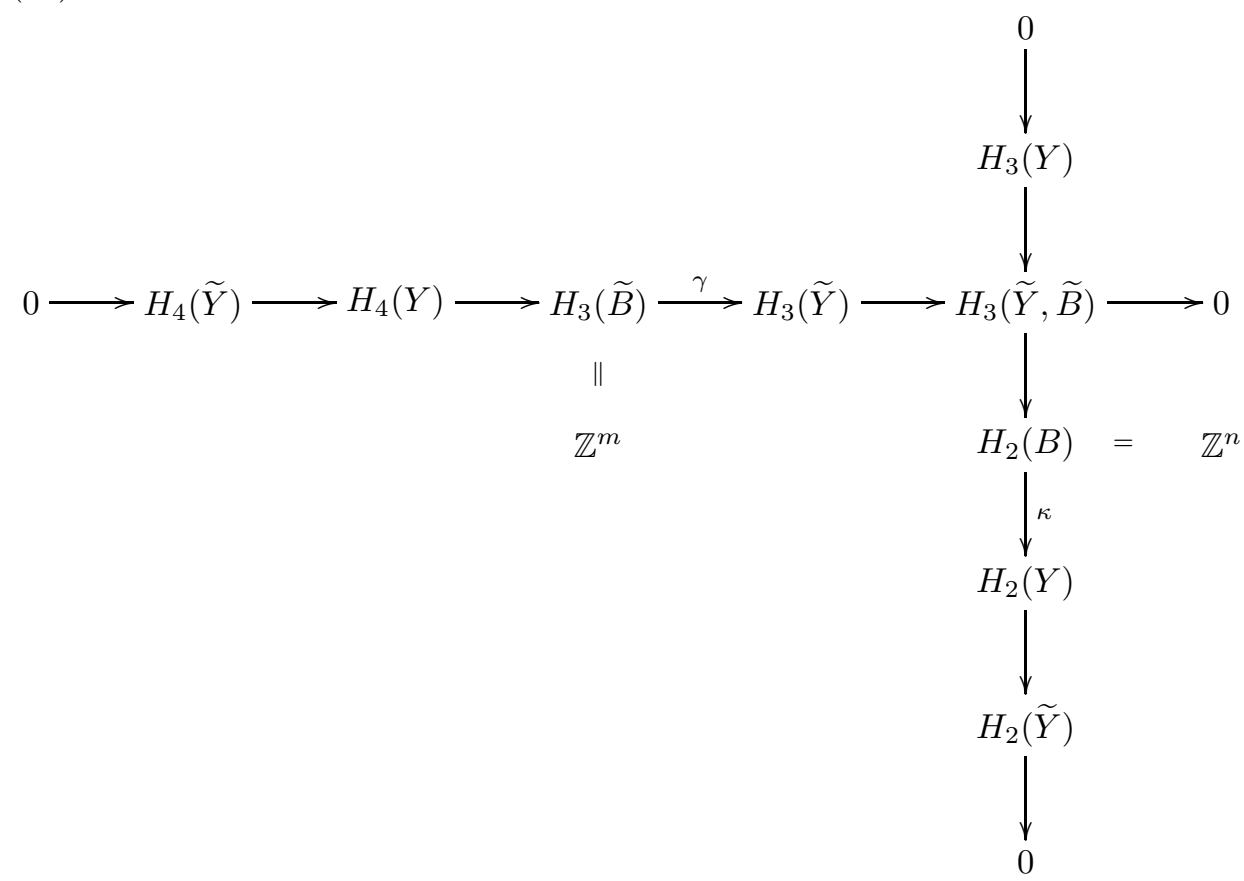


Set

$$
I:=\operatorname{Im}\left[\kappa: \mathbb{Z}^{n}=H_{2}(B) \longrightarrow H_{2}(Y)\right]
$$

Then $k:=\operatorname{rk}(I)$ is the number of linear independent classes of exceptional curves in $H_{2}(Y)$. Since

$$
0 \longrightarrow \mathrm{I} \longrightarrow \mathrm{H}_{2}(Y) \longrightarrow \mathrm{H}_{2}(\widetilde{Y}) \longrightarrow 0
$$

is a short exact sequence, it follows that

$$
b_{2}(Y)=b_{2}(\tilde{Y})+k
$$

On the other hand set

$$
K:=\operatorname{ker}\left[\gamma: \mathbb{Z}^{m} \cong H_{3}(\widetilde{B}) \longrightarrow H_{3}(\widetilde{Y})\right]
$$

Then $m-c^{\prime \prime}:=\operatorname{rk}(K)$ is the number of linear independent relations on the classes of vanishing cycles in $H_{3}(\tilde{Y})$. Since

$$
0 \longrightarrow H_{4}(\widetilde{Y}) \longrightarrow H_{4}(Y) \longrightarrow K \longrightarrow 0
$$

is a short exact sequence, it follows that

$$
b_{4}(Y)=b_{4}(\tilde{Y})+m-c^{\prime \prime}
$$

Conclusion. (i) follows from Step IV and the definition of $n$ and $c^{\prime}$.

(ii) follows from Steps III and IV.

Finally (a), (b) and (c) of Definition 1 follow from Steps I, II, III and IV.

The last assertion of the statement follows from the fact that, if $p$ is a node, then $m_{p}=n_{p}=1$.

Remark 4.6. Notice that Step IV in the proof of Theorem 4.3 gives the following homological interpretation of $k, c^{\prime}, c^{\prime \prime}$

$$
k=\operatorname{rk}(\operatorname{Im} \kappa) \quad, \quad c^{\prime}=\operatorname{rk}(\operatorname{ker} \kappa) \quad, \quad c^{\prime \prime}=\operatorname{rk}(\operatorname{Im} \gamma),
$$

where $\kappa$ and $\gamma$ are the homonymous maps in diagram (25).

Compare with the case of a type II g.t. whose exceptional locus is a del Pezzo surface of degree $d \leq 4$, treated in the following Remark [5.5.

\section{Homological tyPe OF tYPE II GeOMETRIC tRAnSition}

In the present section we will consider a type $I I$ g.t.

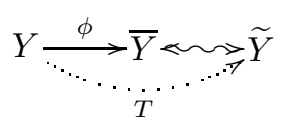

Recalling Theorem[1.9] and Definition[1.10, $\phi$ turns out to be a primitive contraction of an irreducible divisor $E \subset Y$ down to a point $p \in \bar{Y}$. Let us summarize what is known. 
5.0.1. About the singularity $p=\phi(E)$. It is a canonical singularity and in particular it is a rational Gorenstein singular point. The Reid invariant $\varrho$ of $p$ (see [39]) can assume every value $1 \leq \varrho \leq 8$ and

- for $\varrho \leq 3, p$ is a i.h.s.;

- for $\varrho \geq 4, p$ has multiplicity $\varrho$ and minimal embedding dimension

$$
\operatorname{dim}\left(m_{p} / m_{p}^{2}\right)=\varrho+1 ;
$$

in particular, for $\varrho=4, p$ is a complete intersection singularity and, for $\varrho \geq 5, p$ is never a complete intersection singular point (see 5.1 .4 below);

5.0.2. About the exceptional locus E of $\phi$. It is a generalized del Pezzo surface (see [39], Proposition (2.13)) which is:

- either $E$ is a normal del Pezzo surface of degree $1 \leq d=K_{E}^{2} \leq 8$; in particular the degree $d$ equals the Reid invariant $\varrho$ of $p=\phi(E)$;

- or $E$ is a non-normal del Pezzo surface (see [42]).

Observe that the values 0 and 9 cannot be assumed by $\varrho=d$ : the former since $E$ has ample anti-canonical bundle, the latter because (26) is a transition while the contraction of a normal del Pezzo surface of degree 9 down to a point do not admit any smoothing: in this case $E \cong \mathbb{P}^{2}$ and $(\bar{Y}, p)$ is rigid (see [1] and [45]).

Remark 5.1. The contraction $\phi: Y \rightarrow \bar{Y}$ is the (weighted, for $d \leq 2$ ) blow up of the singular point $\phi(p)$ (see [39], Theorem (2.11)). Then $\bar{Y}$ is always $\mathbb{Q}-$ factorial, by [23, Proposition 5-1-6.

5.1. Normal exceptional divisor. A normal del Pezzo surface $E$ occurring as exceptional locus of $\phi$ in (26) is a normal projective Gorenstein surface with ample anti-canonical bundle. Let $\pi: \widehat{E} \rightarrow E$ be a minimal resolution of $E$. The following results are essentially due to F. Hidaka and K.-I. Watanabe [18, M. Reid [39, H. Pinkham [37] and C.Tamás [50].

5.1.1. (18 Proposition 2.1 and Theorem 2.2) $E$ is birationally equivalent to a ruled surface and

- either $E$ is rational,

- or $\widehat{E}$ is a $\mathbb{P}^{1}$-bundle over an elliptic curve; under the notation introduced in [18, we will say that $E$ is elliptic.

In particular $H^{1}\left(E, \mathcal{O}_{E}\right)=0$ ([18, Corollary 2.5).

5.1.2. Let $E$ be rational. Then $E$ can assume at worst isolated Du Val singularities. Moreover (see [18, Theorem 3.4):

- if $d=8$ then either $E \cong \mathbb{P}^{1} \times \mathbb{P}^{1}$ or $E$ is the cone over a conic in $\mathbb{P}^{2}$; in the latter case $\widehat{E} \cong \mathbb{P}(\mathcal{O} \oplus \mathcal{O}(-2))$ and $\pi$ is the contraction of the minimal section of $\widehat{E}$ (we have excluded the case $E \cong \mathbb{P}(\mathcal{O} \oplus \mathcal{O}(-1))$ since the contraction $\phi$ yields a rigid singularity $(\bar{Y}, \phi(E))$, contradicting diagram $(26)$ );

- if $1 \leq d \leq 7$ then there exists a set $\Sigma$ of points on $\mathbb{P}^{2}$ in almost general position (see [18, Definition 3.2) such that $|\Sigma|=9-d$ and $\widehat{E}$ is the blow up of $\mathbb{P}^{2}$ along $\Sigma ; \pi$ is the contraction of all curves on $\widehat{E}$ with self-intersection number -2 . 
5.1.3. If $E$ is rational then $|\operatorname{Sing}(E)| \leq 6$ (see [18, Theorem 4.9, 37] and [50] subsection 3.2).

5.1.4. If $d \geq 4$ then $E$ is rational (15 Thm. (5.2)). Moreover, the anti-canonical map embeds $E$ in $\mathbb{P}^{d}$ as a surface of degree $d$ obtained by intersecting $d(d-3) / 2$ hyperquadrics ([18], Theorem 4.4(i) and Corollary 4.5(i)).

5.1.5. Let $E$ be elliptic. Then $E$ can assume at worst one elliptic singular point (see [39], Definition (2.4)), $\widehat{E} \cong \mathbb{P}\left(\mathcal{O}_{C} \oplus \mathcal{L}\right)$, where $C$ is a smooth elliptic curve and $\mathcal{L}$ is a positive line bundle on $C$, and $\pi$ is the contraction of the minimal section of $\widehat{E}$ (18], Theorem 2.2). In particular, by [5.1.4, $d=\operatorname{deg} E \leq 3$.

5.1.6. If $d=3$ then the canonical map embeds $E$ as a cubic surface in $\mathbb{P}^{3}([18]$ Theorem 4.4(ii) and Corollary 4.5(i), 39] Proposition (2.3)). Then $E$ is elliptic if and only if it is a cone over a plain cubic curve.

5.1.7. If $d=2$ then $E$ is isomorphic to a degree 4 hypersurface in the weighted projective space $\mathbb{P}(1,1,1,2)$. The divisor $-2 K_{E}$ is very ample and the associated morphism embeds $E$ as a degree 8 subvariety of $\mathbb{P}^{6}$. Moreover, $E$ can be described as a double covering of $\mathbb{P}^{2}$ ramified along a quartic curve without multiple components (see 5.4.1 for $d=2$ ). Then $E$ is elliptic if and only if the ramification divisor is given by four lines meeting in a point([18] Theorem 4.4(iii), Corollary 4.5(ii) and Proposition 4.6(i), 39] Proposition (2.3)).

5.1.8. If $d=1$ then $E$ is isomorphic to a degree 6 hypersurface in the weighted projective space $\mathbb{P}(1,1,2,3)$. The divisor $-3 K_{E}$ is very ample and the associated morphism embeds $E$ as a degree 9 subvariety of $\mathbb{P}^{6}$. Moreover, $E$ can be described as a double covering of a quadratic cone $\mathcal{C} \subset \mathbb{P}^{3}$, ramified along the intersection $\mathcal{C} \cap S$, where $S$ is a cubic surface without multiple components and not containing the vertex of the cone (see 5.4.1 for $d=1$ ). Then $E$ is elliptic if and only if the cubic surface $S$ is given by three planes meeting in a line which is tangent to $\mathcal{C}$ ([18] Theorem 4.4(iv), Corollary 4.5(iii) and Proposition 4.6(ii), 39] Proposition (2.3)).

5.2. Non-normal exceptional divisor. A non-normal del Pezzo surface $E$ occurring as exceptional locus of $\phi$ in (26) has to satisfy the classification given in 42. On the other hand $E$ is an irreducible surface embedded in the smooth Calabi-Yau 3-fold $Y$, meaning that $E$ cannot admit non-hypersurface singularities. M. Gross proved that these conditions imply one and only one of the following statements

(i) Consider the Segre-del Pezzo scroll $\mathbb{F}_{a}:=\mathbb{P}\left(\mathcal{O}_{\mathbb{P}^{1}} \oplus \mathcal{O}_{\mathbb{P}^{1}}(-a)\right)$ embedded in $\mathbb{P}^{a+5}$ by means of the very ample linear system $\left|C_{0}+(a+2) f\right|$, where $C_{0}$ is the class of a section and $f$ the class of a fiber. Then $E$ is the projection of $\mathbb{F}_{a}$ into $\mathbb{P}^{a+4}$ from a point in the plane spanned by the conic $C_{0}$ but not on $C_{0}$. This projection exhibits $C_{0}$ as a double covering of a line and makes no other identifications.

(ii) Consider $\mathbb{F}_{a}$ embedded in $\mathbb{P}^{a+3}$ by means of the very ample linear system $\left|C_{0}+(a+1) f\right|$. Then $E$ is the projection of $\mathbb{F}_{a}$ into $\mathbb{P}^{a+2}$ from a point in the plane spanned by the line $C_{0}$ and one fiber $f$. This projection identifies $C_{0}$ and $f$.

In particular, a non-normal $E$ can occur only if $\operatorname{deg}(E)$, or equivalently the Reid invariant $\varrho$ of $p=\phi(E)$, is equal to 7 ([15], Theorem 5.2). 
5.3. Homology change induced by a type II geometric transition. Consider a type II g.t. (26). Then $\phi$ is a primitive (in case weighted) blow up of an isolated 3-dimensional rational Gorenstein singular point $p \in \bar{Y}$, whose exceptional locus is an irreducible generalized del Pezzo surface $E \subset Y$. We get then the following result, which is the type II analogue of Theorems 3.1 and 4.3 .

Theorem 5.2. The type II g.t. (26) admits homological type

$$
h[T]=\left(0,1, c^{\prime}, c^{\prime \prime}\right)
$$

given by

(i) $c^{\prime}=-1+b_{2}(E)-b_{3}(E)$,

(ii) $c^{\prime \prime}=1-\chi(\widetilde{B})=m_{p}-b_{2}(\widetilde{B})$,

where $\widetilde{B}$ is the Milnor fiber of the smoothing $\widetilde{Y}$ and $m_{p}:=b_{3}(\widetilde{B})$ is the Milnor number of the unique singular point $p=\phi(E) \in \bar{Y}$. In particular $b_{1}(\widetilde{B})=0$.

Proof. Let us start by proving (c) in Definition 1, with

$$
k=1=k^{\prime \prime} \quad, \quad c^{\prime}=\chi(E)-3 \quad, \quad c^{\prime \prime}=1-\chi(\widetilde{B}) .
$$

Consider the same notation introduced in the proof of Theorem 4.3, but observe that now $P:=\operatorname{Sing}(\bar{Y})=\{p\}$. Then the singular homology long exact exact sequences of the Mayer-Vietoris couples $\mathcal{C}$ and $\overline{\mathcal{C}}$ are given by

$$
\cdots \longrightarrow H_{q}\left(U^{*}\right) \longrightarrow H_{q}\left(Y^{*}\right) \oplus H_{q}\left(U_{p}\right) \longrightarrow H_{q}(Y) \longrightarrow H_{q-1}\left(U^{*}\right) \longrightarrow \cdots
$$

$$
\cdots \longrightarrow H_{q}\left(\bar{U}^{*}\right) \longrightarrow H_{q}\left(\bar{Y}^{*}\right) \oplus H_{q}\left(\bar{U}_{p}\right) \longrightarrow H_{q}(\bar{Y}) \longrightarrow H_{q-1}\left(\bar{U}^{*}\right) \longrightarrow \cdots
$$

Then Theorem 2.3 and Proposition 2.4 give that

$$
H_{q}\left(\bar{U}_{p}\right) \cong H_{q}(p) \cong\left\{\begin{array}{cc}
\mathbb{Z} & \text { if } q=0 \\
0 & \text { otherwise }
\end{array}\right.
$$

On the other hand Proposition 2.7 gives a strong deformation retraction $U_{p} \approx B \approx$ $E$, giving

$$
\forall q \quad H_{q}\left(U_{p}\right) \cong H_{q}(B) \cong H_{q}(E) .
$$

Then the first equation in Definition 1. (c) follows by comparing (28) and (29).

Consider now the singular homology long exact sequence of the Mayer-Vietoris couple $\widetilde{\mathcal{C}}$

$$
\cdots \longrightarrow H_{q}\left(\widetilde{U}^{*}\right) \longrightarrow H_{q}\left(\widetilde{Y}^{*}\right) \oplus H_{q}\left(\widetilde{U}_{p}\right) \longrightarrow H_{q}(\widetilde{Y}) \longrightarrow H_{q-1}\left(\widetilde{U}^{*}\right) \longrightarrow \cdots
$$

Proposition 2.4 and Theorem 2.5 then guarantee that

$$
\forall q \quad H_{q}\left(\widetilde{U}_{p}\right) \cong H_{q}(\widetilde{B}) \Rightarrow \forall q \geq 4 \quad b_{q}\left(\widetilde{U}_{p}\right)=0 .
$$

Comparing (29) and (32) one gets the second relation in Definition 1.(c).

Assume now the first equation of (a) in Definition 1 giving $b_{2}(\bar{Y})=b_{2}(\tilde{Y})$ and notice that $b_{1}(\tilde{Y})=b_{1}(Y)=0$, since $Y$ and $\tilde{Y}$ are Calabi-Yau 3 -folds. The latter gives in particular that $b_{1}(\bar{Y})=0$, as a consequence of the Leray spectral sequence of the sheaf $\phi_{*} \mathbb{Z}$. Then the comparison of (29) to (32) and the Five Lemma give $H_{1}(\widetilde{B}) \cong H_{1}(P)=0$, proving (ii). 
The proof of (a) and (b) in Definition 1, with $k=1$ and $c^{\prime}, c^{\prime \prime}$ as in the statement, are postponed to subsections 5.4 5.5 and 5.6 where these relations will be analyzed, case by case, under stronger assumptions. In particular, (i) follows by (34), (65) and (69).

5.4. The normal and rational case. Given the type II g.t. (26) let us now consider the case in which $E$ is assumed to be a normal and rational del Pezzo surface. This hypothesis includes all the cases in which $E$ is smooth and it is not so restrictive since it leaves out only the following two further cases:

- $E$ is a normal and elliptic del Pezzo surface,

- $E$ is a non-normal del Pezzo surface,

which will be discussed in the following subsections 5.5 and 5.6, respectively.

Theorem 5.3. Assume that the type II g.t. (26) admits a normal and rational del Pezzo surface as exceptional divisor $E=\operatorname{Exc}(\phi)$ and let $\pi: \widehat{E} \rightarrow E$ be a minimal resolution of $E$, with $L:=\operatorname{Exc}(\pi)$ which is composed by $n_{E}$ rational curves organized in at most six $A-D-E$ trees, by 5.1.2 and 5.1.3. Then it admits homological type $h[T]=\left(0,1, c^{\prime}, c^{\prime \prime}\right)$ as in Theorem 5.2 and moreover

(i) $c^{\prime}=9-d-n_{E}$,

(ii) if $d \leq 4$ then $c^{\prime \prime}=m_{p}$.

Let us postpone the proof of the previous theorem to recall that $\bar{Y}$ is $\mathbb{Q}$-factorial, by Remark 5.1. This fact allows us to conclude the following

Lemma 5.4. Let $p \in \bar{Y}$ be the 3-dimensional i.s. obtained as image of a (generalized) del Pezzo surface $E \subset Y$ under a type II birational contraction from a Calabi-Yau threefold $Y$, as above. Then

$$
b_{4}(\bar{Y})=b_{2}(\bar{Y}) .
$$

Proof. If $p \in \bar{Y}$ is an i.h.s. then the statement is a consequence of Lemmas (3.3) and (3.5) in [36. Actually we will observe that Namikawa-Steenbrink considerations applies to the more general case of $p \in \bar{Y}$, too.

Following their notation, notice first of all that, since $p \in \bar{Y}$ is a rational singular point, $W e i l(\bar{Y}) / \operatorname{Cart}(\bar{Y})$ is a finitely generated abelian group and let $\sigma(\bar{Y})$ denote its rank. Moreover, the rationality of $p$ gives $h^{2}\left(\mathcal{O}_{\bar{Y}}\right)=0$, by Leray spectral sequence. Then Lemma (3.5) in [36] applies to $p \in \bar{Y}$ : in fact arguments proving this Lemma are local cohomology exact sequence, Leray spectral sequence and GoreskyMacPherson theorem 47 Theorem (1.11) holding for an i.s. By applying Lemma (3.3) in [36] we get that $\sigma(\bar{Y})=b_{2}(\bar{Y})-b_{4}(\bar{Y})$. Then $\mathbb{Q}$-factoriality of $\bar{Y}$ ends up the proof.

Proof of Theorem 5.3, By 5.1.2 and 5.1.3, $E=\operatorname{Exc}(\phi)$ admits at worst six isolated Du Val singularities. Moreover, a minimal resolution $\pi: \widehat{E} \rightarrow E$ is the contraction of all curves in $\widehat{E}$ with self-intersection number -2 where $\widehat{E}$ is either $\mathbb{P}^{1} \times \mathbb{P}^{1}$ (when $d=8$ ) or $\mathbb{P}^{2}$ blown up in $9-d$ points in almost general position. Define $Q:=\operatorname{Sing}(E)$ and consider the exceptional tree $L_{q}:=\pi^{-1}(q)$, for any $q \in Q$. Saying $n_{q}$ the number of irreducible components of $L_{q}$ consider the global number $n_{E}:=\sum_{q \in Q} n_{q}$. The same induction argument proving (18) gives $n_{E}=b_{2}(L)$. Consider the relative homology of the couple $(\widehat{E}, L)$ and define $\kappa_{E}: H_{2}(L, \mathbb{Q}) \rightarrow$ 
$H_{2}(\widehat{E}, \mathbb{Q})$. Call

$$
k_{E}:=\operatorname{rk}\left[\operatorname{Im}\left(\kappa_{E}\right)\right] \quad, \quad c_{E}:=\operatorname{rk}\left[\operatorname{ker}\left(\kappa_{E}\right)\right] .
$$

Then $n_{E}=k_{E}+c_{E}$ and we claim that

$$
b_{0}(E)=b_{4}(E)=1, b_{1}(E)=0, b_{3}(E)=c_{E}, b_{2}(E)=10-d-k_{E} .
$$

In fact, first equalities on the left of (34) are clearly obvious. Then apply the local analysis in $\$ 2$ to any $q \in Q \subset E$ and define

$$
\begin{array}{llll}
U_{Q}:=\bigcup_{q \in Q} U_{q} \quad, \quad B_{Q}:=\bigcup_{q \in Q} B_{q} \quad, \quad \widehat{E}^{*}:=\widehat{E} \backslash B_{Q} \quad, \quad U_{Q}^{*}:=U_{Q} \backslash B_{Q} ; \\
\bar{U}_{Q}:=\bigcup_{q \in Q} \bar{U}_{q} \quad, \quad \bar{B}_{Q}:=\bigcup_{q \in Q} \bar{B}_{q} \quad, \quad E^{*}:=E \backslash \bar{B}_{Q} \quad, \quad \bar{U}_{Q}^{*}:=\bar{U}_{Q} \backslash \bar{B}_{Q} .
\end{array}
$$

Then compare the associated Mayer-Vietoris homology sequences:

$$
\cdots \longrightarrow H_{i}\left(U_{Q}^{*}\right) \longrightarrow H_{i}\left(\widehat{E}^{*}\right) \oplus H_{i}\left(U_{Q}\right) \longrightarrow H_{i}(\widehat{E}) \longrightarrow H_{i-1}\left(U_{Q}^{*}\right) \longrightarrow \cdots
$$

$$
\cdots \longrightarrow H_{i}\left(\bar{U}_{Q}^{*}\right) \longrightarrow H_{i}\left(E^{*}\right) \oplus H_{i}\left(\bar{U}_{Q}\right) \longrightarrow H_{i}(E) \longrightarrow H_{i-1}\left(\bar{U}_{Q}^{*}\right) \longrightarrow \cdots
$$

Going on precisely as in Step I of the Proof of the Theorem 4.3 and recalling that $\widehat{E}$ is either $\mathbb{P}^{1} \times \mathbb{P}^{1}$ or the blow up of $\mathbb{P}^{2}$ in $9-d$ in almost general position, one gets the following relation

$$
b_{4}(\widehat{E})-n_{E}+10-d=b_{4}(E)-b_{3}(E)+b_{2}(E)-b_{1}(E) .
$$

On the other hand, compare the relative homology sequences of the couples $(E, Q)$ and $(\widehat{E}, L)$ as follows

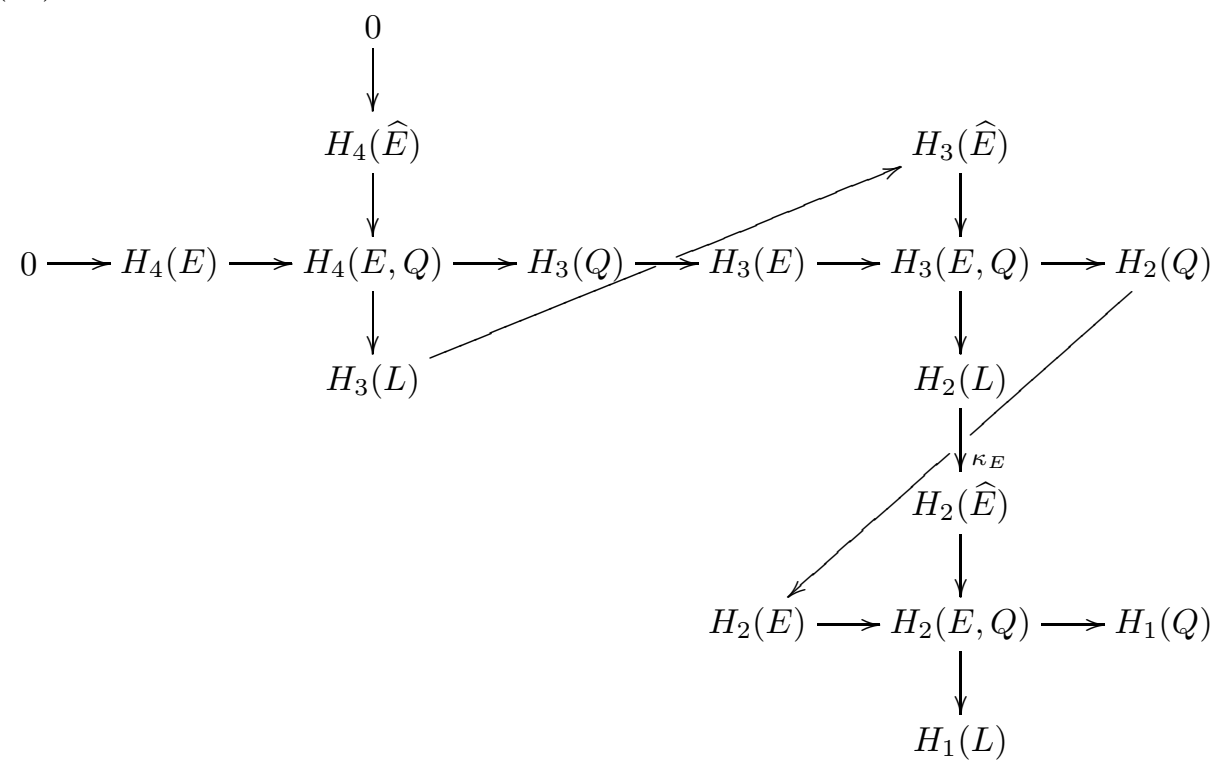

Observe that $b_{3}(L)=b_{1}(L)=b_{3}(Q)=b_{2}(Q)=b_{1}(Q)=0$. Hence $H_{4}(E) \cong$ $H_{4}(E, Q) \cong H_{4}(\widehat{E})$, giving

$$
b_{4}(\widehat{E})=b_{4}(E)
$$


and $H_{i}(E) \cong H_{i}(E, Q)$ for $i=2,3$. Then the right vertical sequence in (38) translates immediately in the following exact sequence

$$
0 \longrightarrow H_{3}(\widehat{E}) \longrightarrow H_{3}(E) \longrightarrow H_{2}(L) \stackrel{\kappa_{E}}{\longrightarrow} H_{2}(\widehat{E}) \longrightarrow H_{2}(E) \longrightarrow 0
$$

Recalling that $b_{2}(L)=n_{E}, b_{3}(\widehat{E})=0$ and $b_{2}(\widehat{E})=10-d$ we get

$$
b_{3}(E)-b_{2}(E)=n_{E}-10+d .
$$

The comparison of (37), (39) and (41) then gives $b_{1}(E)=0$.

Moreover, $b_{3}(\widehat{E})=0$ in (40) gives that $b_{3}(E)=\operatorname{rk}\left[\operatorname{ker}\left(\kappa_{E}\right)\right]=c_{E}$.

The last equality in (34) is then obtained by recalling that $n_{E}=k_{E}+c_{E}$.

Let us now prove relations in Definition 1.(a). First of all recall Mayer-Vietoris exact sequences (28), (29) and (32) of couples $\mathcal{C}, \overline{\mathcal{C}}$ and $\widetilde{\mathcal{C}}$. Relations (34) and the Five Lemma prove that

$$
b_{1}(Y)=b_{1}(\bar{Y}) \quad, \quad b_{5}(\bar{Y})=b_{5}(\tilde{Y}) .
$$

But $b_{1}(Y)=b_{1}(\tilde{Y})=0$, since $Y$ and $\tilde{Y}$ are Calabi-Yau threefolds. Then (42) and Poincaré duality prove the first line in Definition 11.(a).

For the second line in Definition 1.(a), observe that Lefschetz duality and Ehresmann Fibration Theorem give

$$
H_{i}(Y, B) \cong H^{6-i}\left(Y^{*}\right) \cong H^{6-i}\left(\widetilde{Y}^{*}\right) \cong H_{i}(\widetilde{Y}, \widetilde{B}) .
$$

Then isomorphisms (31) and the vanishing in (34) reduce relative homology long exact sequences of couples $(Y, B)$ and $(\widetilde{Y}, \widetilde{B})$ to give the following type II version of diagram (25), where vertical sequences are given by relative homology of couple $(Y, B)$, while horizontal ones are obtained by relative homology of $(\widetilde{Y}, \widetilde{B})$ :

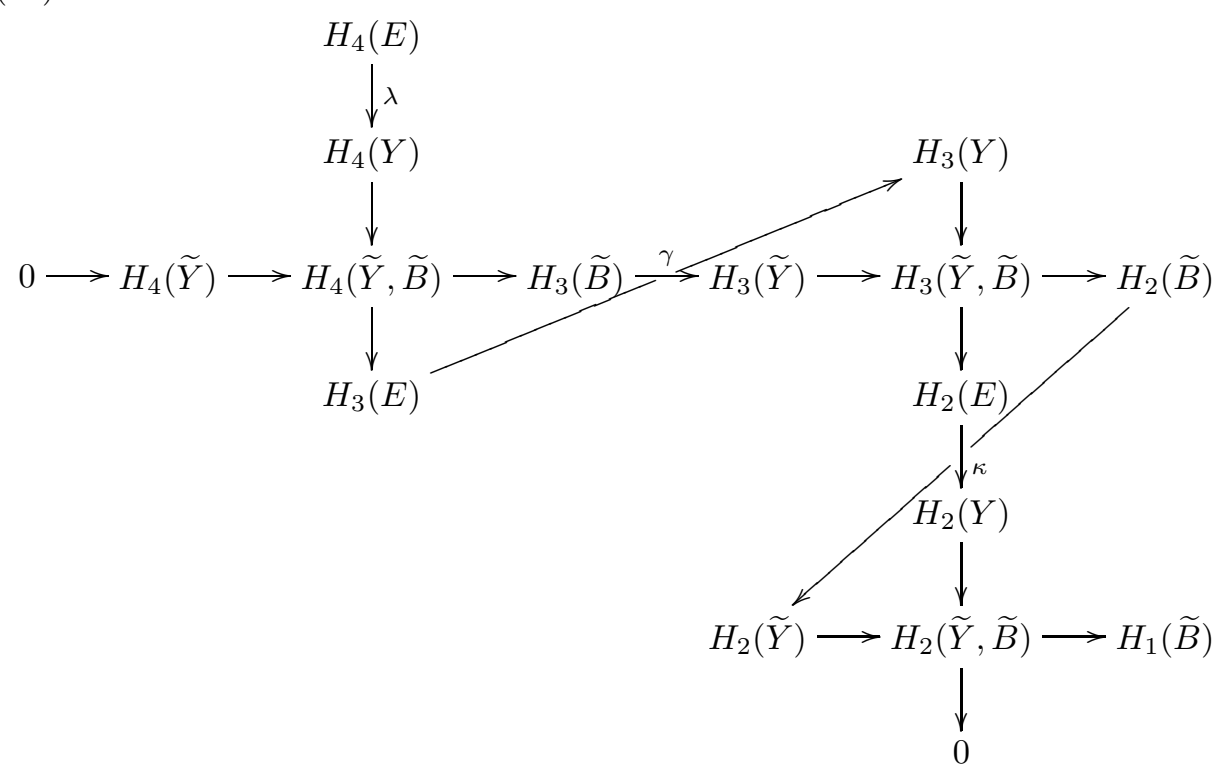

The vertical sequence on the left of this diagram gives the following short exact sequence

$$
0 \longrightarrow \operatorname{Im}(\lambda) \longrightarrow H_{4}(Y) \longrightarrow H_{4}(\tilde{Y}, \widetilde{B}) \longrightarrow 0
$$


MICHELE ROSSI

Observe that $b_{4}(E)=1$ and $E \subset Y$ is the exceptional divisor of a blow up, giving $\operatorname{rk}(\operatorname{Im} \lambda)=1$. Therefore

$$
b_{4}(Y)-b_{4}(\widetilde{Y}, \widetilde{B})=1 .
$$

Since $\tilde{Y}$ is a Calabi-Yau threefolds, $h^{1}\left(\mathcal{O}_{\tilde{Y}}\right)=h^{2}\left(\mathcal{O}_{\tilde{Y}}\right)=0$ and the exponential sequence gives $\operatorname{Pic}(\widetilde{Y}) \cong H^{2}(\widetilde{Y}, \mathbb{Z})$. On the other hand, by [25] (12.2.1.3) and (12.2.1.4.2), we are in a position to apply Proposition 3.1 in [16] implying that the Picard number remains invariant when smoothing $\bar{Y}$, which is

$$
\rho(\bar{Y})=\rho(\tilde{Y})=b_{4}(\tilde{Y}) .
$$

Since $p \in \bar{Y}$ is a rational singularity, pushing forward the exponential sequence for $Y$ induces the following exact sequence on $\bar{Y}$

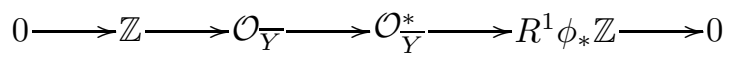

On the other hand, the Leray spectral sequence converging to $H^{i}(Y, \mathbb{Z})$ gives the following lower terms exact sequence

$0 \longrightarrow H^{1}(\bar{Y}, \mathbb{Z}) \longrightarrow H^{1}(Y, \mathbb{Z}) \longrightarrow H^{0}\left(\bar{Y}, R^{1} \phi_{*} \mathbb{Z}\right) \longrightarrow H^{2}(\bar{Y}, \mathbb{Z}) \longleftrightarrow H^{2}(Y, \mathbb{Z})$

where the latter injection is given by the fact that $\phi: Y \rightarrow \bar{Y}$ is a blow up centered in a point. Moreover, $Y$ is a Calabi-Yau threefold, giving

$$
H^{1}(Y, \mathbb{Z})=0 \Longrightarrow H^{1}(\bar{Y}, \mathbb{Z})=0 .
$$

Notice that $R^{1} \phi_{*} \mathbb{Z}$ is a skyscraper sheaf supported on $p \in \bar{Y}$, then

$$
H^{0}\left(R^{1} \phi_{*} \mathbb{Z}\right)=0 \quad \Longleftrightarrow \quad R^{1} \phi_{*} \mathbb{Z}=0,
$$

implying that (46) actually gives the following exponential sequence for $\bar{Y}$

$$
0 \longrightarrow \mathbb{Z} \longrightarrow \mathcal{O}_{\bar{Y}} \longrightarrow \mathcal{O}_{\bar{Y}}^{*} \longrightarrow 0
$$

On the other hand, the Leray spectral sequence converging to $H^{i}\left(Y, \mathcal{O}_{Y}\right)$ gives the following lower terms exact sequence

$$
0 \longrightarrow H^{1}\left(\mathcal{O}_{\bar{Y}}\right) \longrightarrow H^{1}\left(\mathcal{O}_{Y}\right) \longrightarrow H^{0}\left(\bar{Y}, R^{1} \phi_{*} \mathcal{O}_{Y}\right) \longrightarrow H^{2}\left(\mathcal{O}_{\bar{Y}}\right) \longrightarrow H^{2}\left(\mathcal{O}_{Y}\right)
$$

where $h^{1}\left(\mathcal{O}_{Y}\right)=h^{2}\left(\mathcal{O}_{Y}\right)=0$ and $R^{1} \phi_{*} \mathcal{O}_{Y}=0$, since $Y$ is a Calabi-Yau threefold and $p \in \bar{Y}$ is a rational singular point. Therefore

$$
h^{1}\left(\mathcal{O}_{\bar{Y}}\right)=h^{2}\left(\mathcal{O}_{\bar{Y}}\right)=0
$$

giving, by (47), that

$$
\operatorname{Pic}(\bar{Y}) \cong H^{1}\left(\mathcal{O}_{\bar{Y}}^{*}\right) \cong H^{2}(\bar{Y}, \mathbb{Z}) .
$$

Recall that $\phi$ is a primitive contraction, which is $\rho(Y)-\rho(\bar{Y})=1$. Then (45), (48), Lemma 5.4 and Poincaré Duality prove the second and the fourth lines in Definition 1.(a) with $k^{\prime}=0$ and $k=k^{\prime \prime}=1$.

Therefore (44) and diagram (43) give that $H_{4}(\widetilde{Y}) \cong H_{4}(\widetilde{Y}, \widetilde{B})$ (guaranteeing the injectivity of $\gamma$ ).

Recall Theorem 5.2 and in particular equations in Definition 1.(c) with $k, c^{\prime}, c^{\prime \prime}$ as in (27). By (34), these equations can be now rewritten as follows

$$
\begin{aligned}
& b_{3}(Y)=b_{3}(\bar{Y})+1-b_{2}(E)+b_{3}(E)=b_{3}(\bar{Y})-9+d+n_{E} \\
& b_{3}(\bar{Y})=b_{3}(\widetilde{Y})+\chi(\widetilde{B})-1,
\end{aligned}
$$


proving relations in the third line of Definition 1. (a) with $c^{\prime}=9-d-n_{E}$, as in point (i) of the statement. Equations in Definition 1,(b) then follow immediately by those in Definition 1. (a) and Calabi-Yau conditions on $Y$ and $\tilde{Y}$.

Let us now assume that $d \leq 4$ : then (ii) is a consequence of Theorem 2.5, since the Milnor fiber $\widetilde{B}$ has the homotopy type of a bouquet of 3 -spheres and

$$
b_{0}(\widetilde{B})=1, b_{1}(\widetilde{B})=b_{2}(\widetilde{B})=0, b_{3}(\widetilde{B})=m_{p} .
$$

Remark 5.5. If the exceptional del Pezzo surface E has degree $d \leq 4$ then $k, c^{\prime}, c^{\prime \prime}$ admit the same homological interpretation given in Remark 4.6 for a small g.t.

In fact, (50) applied to diagram (43) gives $H^{2}(\widetilde{Y}) \cong H^{2}(\widetilde{Y}, \widetilde{B})$ and the following short exact sequence

$$
0 \longrightarrow \operatorname{Im}(\kappa) \longrightarrow H_{2}(Y) \longrightarrow H_{2}(\tilde{Y}) \longrightarrow 0
$$

On the other hand, the second equation in Definition 1,(a) with $k=1$ gives

$$
k=1=b_{2}(Y)-b_{2}(\tilde{Y})=\operatorname{rk}(\operatorname{Im} \kappa) .
$$

Recalling then (i) and (34) we get $k+c^{\prime}=10-d-k_{E}=b_{2}(E)$ giving necessarily that

$$
c^{\prime}=b_{2}(E)-\operatorname{rk}(\operatorname{Im} \kappa)=\operatorname{rk}(\operatorname{ker} \kappa) .
$$

Finally (ii) in Theorem 5.3 and the injectivity of $\gamma$ in diagram (43) imply that

$$
c^{\prime \prime}=\operatorname{rk}(\operatorname{Im}(\gamma)) \text {. }
$$

Remark 5.6. If the exceptional del Pezzo surface $E$ is smooth then, comparing relations (i) in Theorem 5.3 and (ii) in Theorem5.2 with Theorem 3.3 and Remark 3.6 in [22], we get the following conditions on the Milnor number $m_{p}$ of the singular point $p \in \bar{Y}$ :

$$
\begin{aligned}
& \text { if } d=1 \text { then } \quad m_{p}=50 \text {, } \\
& \text { if } d=2 \text { then } \quad m_{p}=27 \text {, } \\
& \text { if } d=3 \text { then } \quad m_{p}=16 \text {, } \\
& \text { if } d=4 \text { then } \quad m_{p}=9 \text {, } \\
& \text { if } d=5 \text { then } \quad m_{p}=b_{2}(\widetilde{B})+4 \text {, } \\
& \text { if } d=6 \text { then } \quad m_{p}=\left\{\begin{array}{c}
b_{2}(\widetilde{B})-1 \\
b_{2}(\widetilde{B})+1
\end{array}\right. \text {, } \\
& \text { if } d=7 \text { then } \quad m_{p}=b_{2}(\widetilde{B}) \\
& \text { if } d=8 \text { then } \quad m_{p}=b_{2}(\widetilde{B})+1
\end{aligned}
$$

Since $p \in \bar{Y}$ is a singular point, (57) and the first case in (56) prove that $b_{2}(\widetilde{B})>0$, showing that the Milnor fiber $\widetilde{B}$ cannot admit the homotopy type of a bouquet of 3-dimensional spheres when $d=7$ and when $d=6$ with $c=1$. 
Notice that $c+k=c+1$ gives the following (dual) Coxeter numbers

$$
c+k=\left\{\begin{array}{cl}
30=\operatorname{Coxeter}\left(E_{8}\right) & \text { for } d=1 \\
18=\operatorname{Coxeter}\left(E_{7}\right) & \text { for } d=2 \\
12=\operatorname{Coxeter}\left(E_{6}\right) & \text { for } d=3 \\
8=\operatorname{Coxeter}\left(D_{5}\right) & \text { for } d=4 \\
5=\operatorname{Coxeter}\left(A_{4}\right) & \text { for } d=5 \\
2=\operatorname{Coxeter}\left(A_{1}\right) & \text { for } d=8
\end{array}\right.
$$

as argued in [33] $\S 7.1$ and in 32 \$3. Unfortunately this is not true for $d=6$ and $d=7$, suggesting a relation between the vanishing of $b_{2}(\widetilde{B})$ and the existence of a Coxeter group whose number gives $c+k$.

5.4.1. Examples. The Reid and Hidaka-Watanabe results (39] Proposition (2.13) and 18 , Theorem 4.4), here reported in 5.1.4, 5.1.6, 5.1.7 and 5.1.8, allows us to easily construct examples of type II g.t's admitting Milnor numbers as in (54), (53), (52) and (51), respectively (see also Theorem 4.5 in 22 for a comparison).

$d=3$. Consider the generic quintic 3 -fold in $\mathbb{P}^{4}$ admitting a triple point in $p:=[1: 0: \cdots: 0] \in \mathbb{P}^{4}$

$$
\bar{Y}:=\left\{x_{0}^{2} f_{3}\left(x_{1}, \ldots, x_{4}\right)+x_{0} f_{4}\left(x_{1}, \ldots, x_{4}\right)+f_{5}\left(x_{1}, \ldots, x_{4}\right)=0\right\},
$$

where $f_{k}$ are homogeneous polynomials of degree $k$.

Clearly the generic quintic 3-fold $\widetilde{Y} \subseteq \mathbb{P}^{4}$ is a smoothing for $\bar{Y}$.

Blow up $\mathbb{P}^{4}$ in $p$ and let $\mathbb{P}^{3}\left[l_{1}: \ldots: l_{4}\right]$ be the exceptional divisor. Then the strict transform $Y$ of $\bar{Y}$ admits exceptional locus given by the following cubic surface $E:=\left\{f_{3}\left(l_{1}, \ldots, l_{3}\right)=0\right\} \subseteq \mathbb{P}^{3}\left[l_{1}: \ldots: l_{4}\right]$, which is smooth for generic $f_{3}$ (compare with [5.1.6). Observe that $Y$ is a Calabi-Yau threefold since $h^{i}\left(\mathcal{O}_{Y}\right)=h^{i}\left(\mathcal{O}_{\bar{Y}}\right)$ (3) Theorem I.(9.1)) and $Y \rightarrow \bar{Y}$ is a crepant resolution (39. Theorem (2.11) and Corollary (2.12)). Therefore

- $T(Y, \bar{Y}, \widetilde{Y})$ is generically a type II g.t. whose exceptional divisor $E$ is a smooth del Pezzo surface of degree 3.

Since $f_{3}=0$ is the local equation of the triple point $p \in \bar{Y}$ and it is a homogeneous polynomial, the Milnor-Orlik Theorem ([29] Theorem 1) allows us to conclude that the Milnor number of $p$ is given by $m_{p}=(3-1)^{4}=16$.

Since $\widetilde{Y}$ is a projective hypersurface, its Betti numbers are well known and Theorem 5.3 gives $c^{\prime}=6, c^{\prime \prime}=16$ and the following table

\begin{tabular}{c|ccc}
\hline \hline & $b_{2}$ & $b_{3}$ & $b_{4}$ \\
\hline & & & \\
$Y$ & 2 & 182 & 2 \\
$\bar{Y}$ & 1 & 188 & 1 \\
$\widetilde{Y}$ & 1 & 204 & 1 \\
\hline \hline
\end{tabular}

$d=2$. Consider the degree 6 hypersurface of $\mathbb{P}(1,1,1,1,2)\left[x_{0}, x_{1}, x_{2}, x_{3}, y\right]$ given by

$$
\bar{Y}:=\left\{x_{0}^{2} f_{4}\left(x_{1}, \ldots, x_{3}, y\right)+x_{0} f_{5}\left(x_{1}, \ldots, x_{3}, y\right)+f_{6}\left(x_{1}, \ldots, x_{3}, y\right)=0\right\},
$$

where $f_{k}$ is a generic weighted homogeneous polynomials of degree $k$. Clearly $p:=[1: 0: \cdots: 0]$ is an i.h.s. for $\bar{Y}$ whose local equation is given by the w.h. polynomial $f_{4}=0$. Then, again by Milnor-Orlik theorem, $m_{p}=(4-1)^{3}=27$. 
The strict transform $Y$ of $\bar{Y}$ under the weighted blow up of $\mathbb{P}(1,1,1,1,2)$ in $p$ admits exceptional locus given by the degree 4 surface $E:=\left\{f_{4}=0\right\} \subseteq \mathbb{P}(1,1,1,2)$ which is smooth for $f_{4}$ sufficiently general.

Finally the generic degree 6 hypersurface of $\tilde{Y} \subset \mathbb{P}(1,1,1,1,2)$ is smooth since the latter has a unique isolated singular point. Then

- $T(Y, \bar{Y}, \widetilde{Y})$ is generically a type II g.t. whose exceptional divisor $E$ is a smooth del Pezzo surface of degree 2.

Observe that $f_{4}\left(x_{1}, x_{2}, x_{3}, y\right)=y^{2}+y g_{2}(\mathbf{x})+g_{4}(\mathbf{x})$ with $g_{l}$ generic homogeneous polynomials of degree $l$ in $\mathbf{x}=x_{1}, x_{2}, x_{3}$. Then $E$ turns out to be a double covering of $\mathbb{P}^{2}[\mathbf{x}]$ ramified along the discriminant quartic plane curve $\Delta=\left\{g_{2}^{2}-4 g_{4}=0\right\}$ (compare with 5.1.7).

Since $\tilde{Y}$ is a weighted projective hypersurface, its Betti numbers are well known ( 8 4.3.2, [48, 20] Thm. 7.2) and Theorem 5.3 gives $c^{\prime}=7, c^{\prime \prime}=27$ and the following table

\begin{tabular}{c|ccc}
\hline \hline & $b_{2}$ & $b_{3}$ & $b_{4}$ \\
\hline & & & \\
$Y$ & 2 & 174 & 2 \\
$\bar{Y}$ & 1 & 181 & 1 \\
$\widetilde{Y}$ & 1 & 208 & 1 \\
\hline \hline
\end{tabular}

$d=1$. Consider the degree 8 hypersurface of $\mathbb{P}(1,1,1,2,3)\left[x_{0}, x_{1}, x_{2}, y, z\right]$ given by

$$
\bar{Y}:=\left\{x_{0}^{2} f_{6}\left(x_{1}, x_{2}, y, z\right)+x_{0} f_{7}\left(x_{1}, x_{2}, y, z\right)+f_{8}\left(x_{1}, x_{2}, y, z\right)=0\right\},
$$

where $f_{k}$ are generic weighted homogeneous polynomials of degree $k$. As before $p:=[1: 0: \cdots: 0]$ is an i.h.s. for $\bar{Y}$ whose local equation is given by the w.h. polynomial $f_{6}=0$. Then $m_{p}=(3-1)(6-1)^{2}=50$.

The strict transform $Y$ of $\bar{Y}$ under the weighted blow up of $\mathbb{P}(1,1,1,2,3)$ in $p$ admits exceptional locus given by the degree 6 surface $E:=\left\{f_{6}=0\right\} \subseteq \mathbb{P}(1,1,2,3)$ which is smooth for $f_{6}$ sufficiently general.

The generic degree 8 hypersurface of $\tilde{Y} \subset \mathbb{P}(1,1,1,2,3)$ is still smooth since the latter has only isolated singular points. Then

- $T(Y, \bar{Y}, \tilde{Y})$ is generically a type II g.t. whose exceptional divisor $E$ is a smooth del Pezzo surface of degree 1.

Observe that $f_{6}\left(x_{1}, x_{2}, y, z\right)=z^{2}+z g_{3}(\mathbf{x}, y)+g_{6}(\mathbf{x}, y)$ with $g_{l}$ generic weighted homogeneous polynomial of degree $l$ in $\mathbf{x}=x_{1}, x_{2}$ and $y$. Then $E$ turns out to be a double covering of $\mathbb{P}[1,1,2]$ ramified along the discriminant degree 6 plane curve $\Delta=\left\{g_{3}^{2}-4 g_{6}=0\right\}$. Let us first of all observe that

$$
g_{3}(\mathbf{x}, y)=h_{1}(\mathbf{x}) \cdot y+h_{3}(\mathbf{x}) \quad, \quad g_{6}(\mathbf{x}, y)=a y^{3}+h_{2}(\mathbf{x}) \cdot y^{2}+h_{4}(\mathbf{x}) \cdot y+h_{6}(\mathbf{x})
$$

where $h_{l}$ is a generic homogeneous polynomials of degree $l$. Then the discriminant curve $\Delta$ do not pass through the unique singular point of $\mathbb{P}[1,1,2]$, which is $[0: 0: 1]$. Moreover, the linear system associated with $\mathcal{O}(2)$ embeds $\mathbb{P}(1,1,2)$ as a quadratic cone $\mathcal{C} \subset \mathbb{P}^{3}$, translating the degree 6 equation of $\Delta$ in the equation of a cubic surface of $\mathbb{P}^{3}$ cutting $\Delta$ on $\mathcal{C}$ outside of its vertex (compare with [5.1.8).

Since $\widetilde{Y}$ is a weighted projective hypersurface, its Betti numbers are well known (8] 4.3.2, 48, 20] Thm. 7.2) and Theorem 5.3 gives $c^{\prime}=8, c^{\prime \prime}=50$ and the following 
table

\begin{tabular}{c|ccc}
\hline \hline & $b_{2}$ & $b_{3}$ & $b_{4}$ \\
\hline & & & \\
$Y$ & 2 & 156 & 2 \\
$\bar{Y}$ & 1 & 164 & 1 \\
$\widetilde{Y}$ & 1 & 214 & 1 \\
\hline \hline
\end{tabular}

$d=4$. Finally let us consider the case of a type II g.t. whose exceptional divisor is a del Pezzo surface of degree 4, meaning that $\operatorname{Sing}(\bar{Y})$ is composed by a unique complete intersection and non-hypersurface singularity. At this purpose consider the degree 6 complete intersection in $\mathbb{P}^{5}$ given by

$$
\bar{Y}:=X_{1} \cap X_{2} \quad \text { with } \quad \begin{aligned}
& X_{1}:=\left\{x_{0} f_{2}(\mathbf{x})+f_{3}(\mathbf{x})=0\right\} \\
& X_{2}:=\left\{x_{0} g_{2}(\mathbf{x})+g_{3}(\mathbf{x})=0\right\}
\end{aligned}
$$

where $f_{k}, g_{k}$ are generic homogeneous polynomials of degree $k$ in $\mathbf{x}=x_{1}, \ldots, x_{5}$. As before $\operatorname{Sing}(\bar{Y})=\{p\}$, where $p:=[1: 0: \ldots: 0]$. In particular, $p$ is a quadratic 3-dimensional c.i.s., meaning that it is locally described by the germ of singularity given by the zero locus in $\mathbb{C}^{5}$ of two homogeneous quadratic polynomials, namely $f_{2}$ and $g_{2}$. Then, recalling [27] Example 1 in (5.11), the Milnor number of $p$ is $m_{p}=2 \cdot 3+3=9$.

Blow up $\mathbb{P}^{5}$ in $p$ and let $\mathbb{P}^{4}[\mathbf{l}]$ be the exceptional divisor. Then the strict transform $Y$ of $\bar{Y}$ admits exceptional locus given by quartic complete intersection surface $E:=\left\{f_{2}(\mathbf{l})=g_{2}(\mathbf{l})=0\right\} \subset \mathbb{P}^{4}[\mathbf{l}]$, which is smooth for $f_{2}, g_{2}$ sufficiently general (compare with 5.1.4).

On the other hand the generic complete intersection $\tilde{Y}$ of two cubic hypersurfaces in $\mathbb{P}^{5}$ is a Calabi-Yau $3-$ fold. Then

- $T(Y, \bar{Y}, \widetilde{Y})$ is generically a type II g.t. whose exceptional divisor $E$ is a smooth del Pezzo surface of degree 4.

Since $\widetilde{Y}$ is a projective complete intersection, its Betti numbers are well known and Theorem 5.3 gives $c^{\prime}=5, c^{\prime \prime}=9$ and the following table

\begin{tabular}{c|ccc}
\hline \hline & $b_{2}$ & $b_{3}$ & $b_{4}$ \\
\hline$Y$ & 2 & 134 & 2 \\
$\bar{Y}$ & 1 & 139 & 1 \\
$\widetilde{Y}$ & 1 & 148 & 1 \\
\hline \hline
\end{tabular}

5.5. The elliptic case. Let us now assume that the type II g.t. (26) admits exceptional locus $E=\operatorname{Exc}(\phi)$ given by a normal and elliptic del Pezzo surface (recall 5.1.1 and 5.1.5). Then Theorem 5.2 can be rewritten as follows:

Theorem 5.7. Assume that the type II g.t. (26) admits a normal and elliptic del Pezzo surface as exceptional divisor $E=\operatorname{Exc}(\phi)$. Then it admits homological type $h[T]=\left(0,1, c^{\prime}, c^{\prime \prime}\right)$ as in Theorem 5.2 and moreover

(i) $b_{2}(E)=1$ and $b_{3}(E)=2$, giving $c^{\prime}=-2$,

(ii) $c^{\prime \prime}=m_{p}$ and in particular it must be even.

The previous (ii) gives immediately the following 
Corollary 5.8. If $\phi: Y \rightarrow \bar{Y}$ is a primitive type II smooth resolution such that $E=\operatorname{Exc}(\phi)$ admits an elliptic singular point and $\bar{Y}$ is smoothable then the Milnor number $m_{p}$ of $p=\phi(E)$ is even.

Example 5.9. The present example is aimed to give an account of how the Milnor number of $p=\phi(E)$ may jump when specializing to an elliptic exceptional del Pezzo surface $E$, respecting Corollary 5.8, Let us, in fact, consider the case $d=2$ in Examples 5.4.1, whose general case gives Milnor number $m_{p}=27$. Specialize to consider

$$
\begin{aligned}
& f_{4}:=y^{2}+g_{2}\left(x_{1}, x_{2}\right) y+g_{4}\left(x_{1}, x_{2}\right) \\
& f_{5}=a x_{3}^{5}+g_{5}\left(x_{1}, x_{2}, y\right) \\
& f_{6}=b x_{3}^{6}+g_{6}\left(x_{1}, x_{2}, y\right)
\end{aligned}
$$

where $g_{k}$ is a generic (weighted, for $k=5,6$ ) homogeneous polynomial of degree $k$ and $(a, b) \neq(0,0)$ : this last condition is necessary to guarantee that

$$
p:=[1: 0: 0: 0: 0] \in \bar{Y} \subset \mathbb{P}:=\mathbb{P}(1,1,1,1,2)
$$

is an isolated singular point. Its local equation in $\mathbb{C}^{4}$ is given by

$$
\begin{aligned}
& y^{2}+g_{2}\left(x_{1}, x_{2}\right) y+g_{4}\left(x_{1}, x_{2}\right)+a x_{3}^{5}=0 \quad \text { if } a \neq 0 \\
& y^{2}+g_{2}\left(x_{1}, x_{2}\right) y+g_{4}\left(x_{1}, x_{2}\right)+b x_{3}^{6}=0 \quad \text { if } a=0 \text { and } b \neq 0 .
\end{aligned}
$$

Milnor-Orlik Theorem (29] Theorem 1) then gives

$$
\begin{array}{ll}
m_{p}=3^{2} \cdot 4=36 & \text { if } a \neq 0 \\
m_{p}=3^{2} \cdot 5=45 & \text { if } a=0 \text { and } b \neq 0 .
\end{array}
$$

The strict transform $Y$ of $\bar{Y}$, under the weighted blow up of $\mathbb{P}$ in $p$, admits as exceptional locus the elliptic del Pezzo surface

$$
E:=\left\{m^{2}+g_{2}\left(l_{1}, l_{2}\right) m+g_{4}\left(l_{1}, l_{2}\right)=0\right\} \subset \mathbb{P}(1,1,1,2)\left[l_{1}, l_{2}, l_{3}, m\right]
$$

admitting an elliptic singular point in $q:=[0: 0: 1: 0] \in \mathbb{P}(1,1,1,2)$. By Corollary 5.8 we see that $Y$ can be a smooth resolution of $\bar{Y}$ only if $a \neq 0$, since only in this case $m_{p}$ is even. In fact, dividing by $l_{3}$, the equations of $\bar{Y}$ in the affine open subset

$$
\mathcal{A}_{0,3}:=\left\{\left(\left[x_{0}: \cdots: x_{3}: y\right],\left[l_{1}, l_{2}, l_{3}, m\right]\right) \in \mathbb{P}(1,1,1,1,2) \times \mathbb{P}(1,1,1,2) \mid\left(x_{0}, l_{3}\right) \neq \mathbf{0}\right\}
$$

are given by $\mathbf{h}=\left(h_{1}, \ldots, h_{4}\right)=\mathbf{0}$ where

$$
\begin{aligned}
& h_{1}:=x_{1}-l_{1} x_{3} \\
& h_{2}:=x_{2}-l_{2} x_{3} \\
& h_{3}:=y-m x_{3}^{2} \\
& h_{4}:=m^{2}+g_{2}\left(l_{1}, l_{2}\right) m+g_{4}\left(l_{1}, l_{2}\right)+a x_{3}+x_{3} g_{5}\left(l_{1}, l_{2}, m\right)+b x_{3}^{2}+x_{3}^{2} g_{6}\left(l_{1}, l_{2}, m\right)
\end{aligned}
$$

whose jacobian in $\mathbf{0} \in \mathcal{A}_{0,3} \cong \mathbb{C}^{7}$ gives

$$
J_{\mathbf{0}}(\mathbf{h}):=\frac{\partial \mathbf{h}(\mathbf{0})}{\partial\left(x_{1}, \ldots, x_{3}, y, l_{1}, l_{2}, m\right)}=\left(\begin{array}{ccccccc}
1 & 0 & 0 & 0 & 0 & 0 & 0 \\
0 & 1 & 0 & 0 & 0 & 0 & 0 \\
0 & 0 & 0 & 1 & 0 & 0 & 0 \\
0 & 0 & a & 0 & 0 & 0 & 0
\end{array}\right) .
$$


Clearly $\operatorname{rk}\left(J_{\mathbf{0}}(\mathbf{h})\right)=4$ if and only if $a \neq 0$, as expected by Corollary 5.8. Observe that if $a \neq 0$ then $T(Y, \bar{Y}, \widetilde{Y})$ is a g.t. whose Betti number can be obtained by Theorem 5.13 giving $c^{\prime}=-2, c^{\prime \prime}=36$ and

\begin{tabular}{c|ccc}
\hline \hline & $b_{2}$ & $b_{3}$ & $b_{4}$ \\
\hline$Y$ & & & \\
$Y$ & 2 & 174 & 2 \\
$\bar{Y}$ & 1 & 172 & 1 \\
$\widetilde{Y}$ & 1 & 208 & 1 \\
\hline \hline
\end{tabular}

Compare with table (60).

5.5.1. Local analysis of the elliptic singularity. The proof of Theorem 5.7 come from the study of a minimal resolution, of a complex smoothing and of the Milnor number of the elliptic singular point of $E$.

Lemma 5.10 (The resolution). Let $\pi: \widehat{E} \rightarrow E$ be a minimal resolution of the elliptic del Pezzo surface E. Then

$$
b_{0}(\widehat{E})=b_{4}(\widehat{E})=1, b_{1}(\widehat{E})=b_{3}(\widehat{E})=2, b_{2}(\widehat{E})=h^{1,1}(\widehat{E})=2 .
$$

Proof. First equations are clearly obvious. By 5.1.5, $\widehat{E} \cong \mathbb{P}\left(\mathcal{O}_{C} \oplus \mathcal{L}\right)$ is a geometrically ruled surface over an elliptic curve $C$. Then

$$
\forall i>0 \quad h^{i}\left(\mathcal{O}_{\widehat{E}}\right)=h^{i}\left(\mathcal{O}_{C}\right) \quad \text { (17] Lemma V.2.4) }
$$

giving $h^{1}\left(\mathcal{O}_{\widehat{E}}\right)=1$ and $h^{2}\left(\mathcal{O}_{\widehat{E}}\right)=0$. These relations and Kodaira-Serre duality suffice to end up the proof.

Lemma 5.11 (The smoothing). Let $\widetilde{E}$ be a smoothing of the elliptic del Pezzo surface $E$. Then

$$
b_{0}(\widetilde{E})=b_{4}(\widetilde{E})=1, b_{1}(\widetilde{E})=b_{3}(\widetilde{E})=0, b_{2}(\widetilde{E})=10-d
$$

where $d=\operatorname{deg}(E)$.

Proof. Recall that $1 \leq d \leq 3$, by 5.1.4. Moreover:

- if $d=3$ then $E$ is described in 5.1.6 and $\widetilde{E}$ is a generic cubic surface in $\mathbb{P}^{3}$,

- if $d=2$ then $E$ is described in 5.1 .7 and $\widetilde{E}$ is a generic quartic surface in $\mathbb{P}(1,1,1,2)$,

- if $d=1$ then $E$ is described in 5.1 .8 and $\widetilde{E}$ is a generic degree 6 surface in $\mathbb{P}(1,1,2,3)$.

Therefore $\widetilde{E}$ turns out to be isomorphic to a smooth degree $d$ del Pezzo surface. Then (62) follow by (34) with $k_{E}=c_{E}=0$.

Lemma 5.12 (The Milnor number). Let $q$ be the unique (elliptic) singular point of an elliptic del Pezzo surface $E$ of degree $\operatorname{deg}(E)=d$. Then the Milnor number of $q \in E$ is given by $m_{q}=11-d$.

Proof. By 5.1.6, if $d=3$ then $q$ is the vertex of a cone over a cubic plane curve $f_{3}\left(x_{1}, x_{2}, x_{3}\right)=0$. Then $m_{q}=2^{3}=8=11-3$.

By 5.1.7 if $d=2$ then $E$ is a double covering of $\mathbb{P}^{2}$ ramified along four distinct lines meeting in the elliptic singular point $q \in E$, whose local equation in $\mathbb{C}^{2}$ is then given the degree 4 homogeneous polynomial in two variables giving the four lines. Then $m_{q}=3^{2}=9=11-2$. 
By 5.1.8, if $d=1$ then $E$ is the double covering of $\mathbb{P}(1,1,2)\left[x_{0}, x_{1}, y\right]$ ramified along the reducible degree 6 curve $\left\{\prod_{i=1}^{3}\left(y-q_{i}\left(x_{1}\right)=0\right\}\right.$, where $\operatorname{deg} q_{i}=2$. The elliptic singular point $q$ is then given by $[1: 0: 0] \in \mathbb{P}(1,1,2)$, whose Milnor number is $m_{q}=2 \cdot 5=10=11-1$, by the Milnor-Orlik Theorem $(29]$ Theorem 1$)$.

5.5.2. Proof of Theorem [5.7. Let us recall that $\pi: \widehat{E} \rightarrow E$ is the contraction of the minimal section of $\widehat{E} \cong \mathbb{P}\left(\mathcal{O}_{C} \oplus \mathcal{L}\right)$ (see 5.1.5). Then setting $\{q\}=Q:=\operatorname{Sing}(E)$ and $L_{q}:=\pi^{-1}(q)$ one has

$$
L=\operatorname{Exc}(\pi)=L_{q} \cong C
$$

and the total number of irreducible components of $L$ is given by $n_{E}=n_{q}=1=$ $b_{2}(L)$ (notation as in the Proof of theorem 5.2). In the present case, (37) has to be rewritten as follows

$$
b_{3}(\widehat{E})+b_{2}(L)-b_{2}(\widehat{E})-b_{1}(L)+b_{1}(\widehat{E})+b_{0}(L)=b_{3}(E)-b_{2}(E)+b_{1}(E)+|Q|
$$

which, by (61) and (63), gives

$$
b_{3}(E)-b_{2}(E)+b_{1}(E)=1 .
$$

On the other hand, calling $\widetilde{A}$ the Milnor fiber near $q \in E$, it has the homotopy type of a bouquet of 2-spheres, by Theorem 2.5 and the fact that $q \in E$ is an isolated hypersurface singularity. By Lefschetz Duality, Ehresmann diffeomorphism and relative homology of the couple $(E, Q)$, one has

$$
\forall i \geq 2 \quad H_{i}(\widetilde{E}, \widetilde{A}) \cong H^{i}(\widetilde{E} \backslash \widetilde{A}) \cong H^{i}(E \backslash Q) \cong H_{i}(E, Q) \cong H_{i}(E)
$$

Then the relative homology long exact sequence of the couple $(\widetilde{E}, \widetilde{A})$ gives the following exact sequence

$$
0 \longrightarrow H_{3}(\widetilde{E}) \longrightarrow H_{3}(E) \longrightarrow H_{2}(\widetilde{A}) \longrightarrow H_{2}(\widetilde{E}) \longrightarrow H_{2}(E) \longrightarrow 0
$$

Apply relations (62) and Lemma 5.12 to such a sequence to get

$$
b_{3}(E)-b_{2}(E)=m_{q}-10+d=1 .
$$

Recall (64) to get then:

$$
b_{0}(E)=b_{4}(E)=1 \quad, \quad b_{1}(E)=0 \quad, \quad b_{3}(E)-b_{2}(E)=1 .
$$

Then point (i) in the statement follows by (65) and observing that the birational contraction $\pi: \widehat{E} \rightarrow E$ is obtained as the contraction of the minimal section of $\widehat{E}$ $\left(\left[18\right.\right.$, Theorem 2.2), whose class is a generator of $H^{2}(\widehat{E}, \mathbb{Q}) \cong \mathbb{Q}^{2}$.

Let us now observe that, by replacing (34) with relations (65), it is still possible to write down equations (42) in the present case, proving equations in the first line in Definition 1.(a). On the other hand, arguments proving (45) and (48) still hold in the present case, proving the second and the fourth line in Definition 1. (a) with $k^{\prime}=0$ and $k=k^{\prime \prime}=1$. Since $\operatorname{deg} E \leq 3$ we can apply relations (50) to equations (49), to get immediately (ii) in the statement and

$$
\begin{aligned}
& b_{3}(Y)=b_{3}(\bar{Y})+1-b_{2}(E)+b_{3}(E)=b_{3}(\bar{Y})+2 \\
& b_{3}(\bar{Y})=b_{3}(\widetilde{Y})+\chi(\widetilde{B})-1=b_{3}(\widetilde{Y})-m_{p},
\end{aligned}
$$

proving the third line in (a), and consequently equations in (b), of Definition 1 with $c^{\prime}, c^{\prime \prime}$ as in (i) and (ii), respectively. 
5.6. The non-normal case. Let us now assume that the type II g.t. (26) admits exceptional locus $E=\operatorname{Exc}(\phi)$ given by a non-normal del Pezzo surface (recall 5.2, cases (i) and (ii)). Then Theorem 5.2 can be rewritten as follows:

Theorem 5.13. Assume that the type II g.t. (26) admits a non-normal del Pezzo surface as exceptional divisor $E=\operatorname{Exc}(\phi)$. Then it admits homological type $h[T]=$ $\left(0,1, c^{\prime}, c^{\prime \prime}\right)$ as in Theorem 5.2 and moreover

(i) $b_{2}(E) \in\{1,2\}$ and $b_{3}(E)=0$, giving $c^{\prime} \in\{0,1\}$, respectively,

(ii) $\chi(\widetilde{B}) \equiv b_{2}(E) \bmod 2$.

Corollary 5.14. If (26) is a type II g.t. with $E=\operatorname{Exc}(\phi)$ a non-normal del Pezzo surface, then $E$ is like in 5.2. (i) (resp. 5.2. (ii)) if and only if $\chi(\widetilde{B})$ is even (resp. odd).

Proof of Theorem 5.13. Let us first of all observe that the normalization $\mathbb{F}_{a}=$ $\mathbb{P}\left(\mathcal{O} \oplus \mathcal{O}_{\mathbb{P}^{1}}(-a)\right)$ of $E$ has the following Betti numbers:

$$
b_{0}\left(\mathbb{F}_{a}\right)=b_{4}\left(\mathbb{F}_{a}\right)=1 \quad, \quad b_{1}\left(\mathbb{F}_{a}\right)=b_{3}\left(\mathbb{F}_{a}\right)=0 \quad, \quad b_{2}\left(\mathbb{F}_{a}\right)=h^{1,1}\left(\mathbb{F}_{a}\right)=2 .
$$

In fact, the first equalities are obvious. Moreover,

$$
\forall i>0 \quad h^{i}\left(\mathcal{O}_{\mathbb{F}_{a}}\right)=h^{i}\left(\mathcal{O}_{\mathbb{P}^{1}}\right)=0
$$

giving the middle equalities in (67) and $b_{2}\left(\mathbb{F}_{a}\right)=h^{1,1}\left(F_{a}\right)$. In particular, $\chi\left(\mathcal{O}_{\mathbb{F}_{a}}\right)=$ 1 and the Noether formula ([3] Theorem I.(5.4)) gives

$$
2+h^{1,1}\left(\mathbb{F}_{a}\right)=\chi\left(\mathbb{F}_{a}\right)=12 \chi\left(\mathcal{O}_{\mathbb{F}_{a}}\right)-K_{\mathbb{F}_{a}}^{2}=4 \Longrightarrow h^{1,1}\left(\mathbb{F}_{a}\right)=2,
$$

where $K_{\mathbb{F}_{a}}=-2 C_{0}-(a+2) f$ is the canonical divisor of $\mathbb{F}_{a}$, giving $K_{\mathbb{F}_{a}}^{2}=8$ (notation as in 5.2, see [17] Corollary V.2.11). Then (i) follows immediately by (67) recalling the construction of the normalization morphism $\mathbb{F}_{a} \rightarrow E$ described in 5.2 (i) and (ii). Moreover, we get

$$
b_{0}(E)=b_{4}(E)=1 \quad, \quad b_{1}(E)=b_{3}(E)=0 \quad, \quad b_{2}(E) \in\{1,2\},
$$

which replaced to (34) guarantee that (42), (45) and (48) still hold, by the same arguments. Then equations in Definition 1. (c), with $k, c^{\prime}, c^{\prime \prime}$ as in Theorem 5.2 , give

$$
\begin{aligned}
& \chi(Y)-\chi(\bar{Y})=2-b_{3}(Y)+b_{3}(\bar{Y})=1+b_{2}(E)=\chi(E)-1 \\
& \chi(\bar{Y})-\chi(\widetilde{Y})=-b_{3}(\bar{Y})+b_{3}(\widetilde{Y})=1-\chi(\widetilde{B})
\end{aligned}
$$

which is enough to prove (a) and (b) in Definition 1 with $k^{\prime}=0, k=k^{\prime \prime}=1$ and $c^{\prime}, c^{\prime \prime}$ assigned by (i) and (ii).

\section{REFERENCES}

[1] Altmann K. "The versal deformation of an isolated toric Gorensteins singularity" Invent. Math. 128(1997), 443-479; math.AG/9403004

[2] Banagl M. "Singular spaces and generalized Poincaré complexes" Electron. Res. Announc. Math. Sci. 16 (2009), 63-73.

[3] Barth W., Peters C. and Van de Ven A. Compact complex surfaces vol. 4 E.M.G, SpringerVerlag (1984)

[4] Batyrev V. "Dual polyhedra and mirror symmetry for Calabi-Yau hypersurfaces in toric varieties" J. Alg. Geom. 3 (1994), 493-535; math.AG/9310003

[5] Candelas P., Horowitz G.T., Strominger A. and Witten E. "Vacuum configurations for superstrings" Nuclear Phys. B258(1) (1985), 46-74.

[6] Clemens C.H. "Double Solids" Adv. in Math. 47 (1983), 107-230.

[7] Cox A.D. and Katz S. Mirror Symmetry and Algebraic Geometry vol. 68 Math. Surveys and Monographs, Amer. Math. Soc., Providence RI (1999). 
[8] Dolgachev I. "Weighted projective varieties" in Group actions and vector fields (Vancouver, B.C., 1981), 34-71, Lecture Notes in Math. 956, Springer, Berlin, 1982.

[9] Enriques F. Le superficie algebriche Zanichelli, Bologna (1946).

[10] Friedman R. "Simultaneous resolution of threefold double points" Math. Ann. 247 (1986), 671-689.

[11] Grassi A. and Rossi M. "Large $N$-dualities and transitions in geometry" in Geometry and Physics of Branes, Como 2001 Series of H.E.P., Cosmology and Gravitation, IoP Bristol (2003), 210-278; math.AG/0209044.

[12] Green P.S. and Hübsch T. "Possible phase transitions among Calabi-Yau compactifications" Phys. Rev. Lett. 61 (1988), 1163-1166.

[13] Green P.S. and Hübsch T. "Connetting moduli spaces of Calabi-Yau threefolds" Comm. Math. Phys. 119 (1988), 431-441.

[14] Greene B., Morrison D.R. and Strominger A. "Black hole condensation and the unification of string vacua" Nucl. Phys. B451 (1995), 109-120; hep-th/9504145.

[15] Gross M. "Deforming Calabi-Yau threefolds" Math. Ann. 308 (1997), 187-220; math.AG/9506022

[16] Gross M. "Primitive Calabi-Yau threefolds" J. Diff. Geom. 45 (1997), 288-318; math.AG/9512002

[17] Hartshorne R. Algebraic Geometry G.T.M. 52, Springer-Verlag, Berlin-Hidelberg-New York (1977).

[18] Hidaka H. and Watanabe K.-I. "Normal Gorenstein surfaces with ample anti-canonical divisor" Tokyo J. Math. 4 (1981), 319-330.

[19] Hirzebruch F. "Some examples of threefolds with trivial canonical bundle" in Collected papers, vol. II, 757-770, Springer (1987).

[20] Iano-Fletcher A.R. "Working with weighted complete intersections" in Explicit birational geometry of 3-folds, London Math. Soc. Lecture Note Ser. 281, Cambridge Univ. Press, Cambridge (2000), 101-173.

[21] Joyce D. Compact manifolds with Special Holonomy, Oxford Science Publications, OxfordNew York (2000).

[22] Kapustka G. and M. "Primitive contractions of Calabi-Yau threefolds. I" Comm. Algebra 37 (2009), 482-502; arXiv:math/0703810v3 [math.AG].

[23] Kawamata Y., Matsuda K. and Matsuki K. "Introduction to the Minimal Model program" Algebraic Geometry, Sendai 1985, Adv. Stud. Pure Math. 10 (1987), 283-360.

[24] Kodaira K. "On the structure of compact complex analytic surfaces" Am. J. Math. 86 (1964), $751-798$.

[25] Kollár J. and Mori S. "Classification of three-dimensional flips" J. Amer. Math. Soc. 5 (1992), 533-703.

[26] Laufer H. "On $\mathbb{C} P^{1}$ as an exceptional set" in Recente developments in several complex variables Ann. Math. Stud. 100 (1981), 261-276.

[27] Looijenga E.J.N. Isolated singular points on complete intersections LMS Lecture Notes 77, Cambridge Univ. Press (1984).

[28] Milnor J. Singular points of complex hypersurfaces, Annals of Math. Studies 61, Princeton University Press, Princeton (1968).

[29] Milnor J. and Orlik P. "Isolated singularities defined by weighted homogeneous polynomials", Topology 9 (1970), 385-393.

[30] Morrison D.R. "The birational geometry of surfaces with rational double points" Math. Ann. 271 (1985), 415-438.

[31] Morrison D.R. "Through the looking glass" in Mirror Symmetry III, American Mathematical Society and International Press (1999), 263-277; math.AG/9705028

[32] Morrison D.R. and Seiberg N. "Extremal transitions and five-dimensional supersymmetric field theories" Nucl. Phys. B483 (1997), 229-247; hep-th/9609070

[33] Morrison D.R.and Vafa C. "Compactifications of F-theory on Calabi-Yau threefolds.II" Nucl. Phys. textbfB476 (1996), 437-469; hep-th/9603161

[34] Miranda R. and Persson U. "On extremal rational elliptic surfaces" Math. Z. 193(4) (1986), $537-558$.

[35] Namikawa Y. "Stratified local moduli of Calabi-Yau 3-folds" Topology 41 (2002), 1219-1237. 
[36] Namikawa Y. and Steenbrink J. "Global smoothing of Calabi-Yau 3-fold" Invent. Math. 122 (1995), 403-419.

[37] Pinkham H. "Simple elliptic singularities, del Pezzo surfaces and Cremona transformations" in Several Complex Variables Proc. Symp. Pure Math. 30 (1977), 69-70.

[38] Pinkham H. "Factorization of birational maps in dimension three" in Singularities Proc. Symp. Pure Math. 40 (1981), 343-372.

[39] Reid M. "Canonical 3-folds" in Journées de géométrie algébrique d'Angers, Sijthoff \& Norddhoff (1980), 671-689.

[40] Reid M. "Minimal model of canonical 3-folds" in Algebraic varieties and analytic varieties, Adv. Stud. Pure Math. 1, North-Holland (1983), 131-180.

[41] Reid M. "The moduli space of 3-folds with $K=0$ may neverthless be irreducible" Math. Ann. 287 (1987), 329-334.

[42] Reid M. "Non-normal del Pezzo surfaces" Math. Proc. RIMS 30 (1994), 695-727.

[43] Rossi M. "Geometric transitions" J. Geom. Phys. 56(9) (2006), 1940-1983; math/0412514.

[44] Rossi M. On a remark by Y.Namikawa arXiv:0807.4104 [math.AG].

[45] Schlessinger M. "Rigidity of quotient singularities" Invent. Math. 14 (1971), 17-26.

[46] Schoen C. "On fiber products of rational elliptic surfaces with section" Math. Z. 197(2) (1988), 177-199.

[47] Steenbrink J.H.M. "Mixed Hodge Structure associated with isolated singularities" in Singularities, Part 2 (Arcata, Calif., 1981), Proc. Sympos. Pure Math. 40 (1983), 513-536.

[48] Steenbrink J.H.M. "Mixed Hodge structures and singularities: a survey" in Géométrie algébrique et applications, III (La Rábida, 1984), Travaux en Cours 24, Hermann, Paris (1987), 99-123.

[49] Strominger A. "Massless black holes and conifolds in string theory" Nucl. Phys. B451 (1995), 97-109; hep-th/9504145

[50] Tamás C. "On the classification of crepant analytically extremal contractions of smooth three-folds" Compos. Math. 140 (2004), 1561-1578; math.AG/0204015

[51] Tian G. "Smoothing threefold with trivial canonical bundle and ordinary double points" in Essays on Mirror Manifolds Internat. Press, Hong Kong (1992), 458-479.

[52] Van Geemen B. and Werner J. "New examples of threefolds with $c_{1}=0$ " Math.Z. 203 (1990), 211-225.

[53] Werner J. "Kleine Auflösungen spezieller dreidimensionaler Varietäten" Bonn. Math. Schr. 186 (1987).

[54] Wilson P.M.H. "The Kähler cone on Calabi-Yau threefolds" Invent.Math. 107 (1992), 561583.

[55] Wilson P.M.H. Erratum to "The Kähler cone on Calabi-Yau threefolds" Invent.Math. 114 (1993), 231-233.

Dipartimento di Matematica, Università di Torino, via Carlo Alberto 10, 10123 TORINO

E-mail address: michele.rossi@unito.it 